\title{
CROSS DIFFUSION AND NONLINEAR DIFFUSION PREVENTING BLOW UP IN THE KELLER-SEGEL MODEL
}

\author{
JOSÉ ANTONIO CARRILLO, SABINE HITTMEIR, AND ANSGAR JÜNGEL
}

\begin{abstract}
A parabolic-parabolic (Patlak-) Keller-Segel model in up to three space dimensions with nonlinear cell diffusion and an additional nonlinear cross-diffusion term is analyzed. The main feature of this model is that there exists a new entropy functional, yielding gradient estimates for the cell density and chemical concentration. For arbitrarily small cross-diffusion coefficients and for suitable exponents of the nonlinear diffusion terms, the global-in-time existence of weak solutions is proved, thus preventing finite-time blow up of the cell density. The global existence result also holds for linear and fast diffusion of the cell density in a certain parameter range in three dimensions. Furthermore, we show $L^{\infty}$ bounds for the solutions to the parabolic-elliptic system. Sufficient conditions leading to the asymptotic stability of the constant steady state are given for a particular choice of the nonlinear diffusion exponents. Numerical experiments in two and three space dimensions illustrate the theoretical results.
\end{abstract}

\section{INTRODUCTION}

Patlak [26] and Keller and Segel [19] have proposed a partial differential equation model, which describes the movement of cells in response to a chemical signal. The cells move towards regions of higher signal concentrations. As the cells produce the signal substance, the movement may lead to an aggregation of cells. The more cells are aggregated, the more the attracting chemical signal is produced by the cells. This process is counter-balanced by cell diffusion, but if the cell density is sufficiently large, the nonlocal chemical interaction dominates and results - in two and three space dimensions - in a blow up of the cell density (see the reviews $[12,15]$ for details). Denoting by $\rho=\rho(x, t)$ the cell density and by $c=c(x, t)$ the concentration of the chemical signal, the Keller-Segel model, in its general

2000 Mathematics Subject Classification. 35K55, 35K65, 35Q80, 78A70, 92C17.

Key words and phrases. Chemotaxis, Keller-Segel model, cross-diffusion, degenerate diffusion, global existence of solutions, blow up.

The authors have been partially supported by the bilateral Austrian-Spanish Project ES 08/2010AT2009-0008 of the Austrian Exchange Service (ÖAD) and MICINN. The work of SH is supported by the King Abdullah University of Science and Technology (KAUST), grant KUK-I1-007-43. SH and AJ acknowledge partial support from the Austrian Science Fund (FWF), grants P20214, P22108, and I395, from the Austrian-French Project FR 07/2010, and from the Austrian-Croatian Project HR 01/2010 of the ÖAD. JAC was partially supported by the Ministerio de Ciencia e Innovación, grant MTM2011-27739C04-02, and by the Agència de Gestió d'Ajuts Universitaris i de Recerca-Generalitat de Catalunya, grant 2009-SGR-345. 
form, is given by

$$
\begin{aligned}
\partial_{t} \rho & =\operatorname{div}(D(\rho) \nabla \rho-\chi(\rho) \nabla c)+R_{1}(\rho, c), \\
\alpha \partial_{t} c & =\Delta c+R_{2}(\rho, S), \quad x \in \Omega, t>0,
\end{aligned}
$$

where $\Omega \subset \mathbb{R}^{d}(d \geq 1)$ is a bounded domain, $D(\rho)$ is the cell diffusivity, $\chi(\rho)$ the chemotactic sensitivity, and $R_{1}(\rho, c)$ and $R_{2}(\rho, c)$ describe the production and degradation of the cell density and chemical substance, respectively. Here, $\alpha=0$ corresponds to the parabolicelliptic case and $\alpha=1$ to the fully parabolic problem. The equations are supplemented by homogeneous Neumann boundary and initial conditions:

$$
\begin{array}{cl}
D(\rho)(\nabla \rho \cdot \nu)=\nabla c \cdot \nu=0 & \text { on } \partial \Omega, t>0, \\
\rho(\cdot, 0)=\rho_{0}, \quad \alpha c(\cdot, 0)=\alpha c_{0} & \text { in } \Omega,
\end{array}
$$

where $\nu$ denotes the exterior unit normal to the boundary $\partial \Omega$ (which is assumed to exist). The classical Keller-Segel model consists in the choice $D(\rho)=1, \chi(\rho)=\rho, R_{1}(\rho, c)=0$, and $R_{2}(\rho, c)=\rho-c$.

Motivated by numerical and modeling issues, the question how blow up of cells can be avoided has been investigated intensively the last years. Up to our knowledge, four methods have been proposed. In the following, we review these methods.

The first idea is to modify the chemotactic sensitivity. Supposing that aggregation stops when the cell density reaches the maximal value $\rho_{\infty}=1$, one may choose $\chi(\rho)=1-\rho$. In this volume-filling case, the cell density is bounded, $0 \leq \rho \leq 1$, and the global existence of solutions can be proved [9]. Furthermore, if $\chi(\rho)=\rho^{\beta}$ with $0<\beta<2 / d$, the solutions are global and bounded, thus preventing finite-time blow up [16]. Global solutions are also obtained when the sensitivity depends on the chemical concentrations in an appropriate way, see, e.g., [1, 13].

A second method consists in modifying the cell diffusion. In the context of the volumefilling effect, Burger et al. [3] suggested the cell equation $\partial_{t} \rho=\operatorname{div}(\rho(1-\rho) \nabla(\rho-c))$. Then the parabolic-elliptic model possesses global solutions. Global existence results can be achieved by employing the nonlinear diffusion $D(\rho)=\rho^{\alpha}$, which models the local repulsion of cells. When $\int_{1}^{\rho}(D(s) / s) d s$ grows faster than log $\rho$ for large $\rho$, a priori estimates showing that solutions are global and uniformly bounded in time were obtained in [4, 21]. Adding the nonlinear sensitivity $\chi(\rho)=\rho^{\beta}$ with $\alpha \geq 1$ and $2 \leq \beta<\alpha+2 / d$, global existence results were achieved in [17]. The solutions are uniformly bounded in time if $\alpha>2-4 / d$ [22]. The existence of global bounded classical solutions to a fast-diffusion Keller-Segel model with $D(\rho)=(1-\rho)^{-\alpha}$, where $\alpha \geq 2$, has been proved in [6]. The same result holds true when we choose $\chi(\rho)=\rho(1-\rho)^{\beta}$ with $\beta \geq 1-\alpha / 2$, and the solution is still global in time (but possibly not classical) if $\beta \geq 1-\alpha[30]$.

A third approach is to consider nonvanishing growth-death models $R_{1} \neq 0$, since one may expect that a suitable death term avoids cell aggregation. Indeed, taking $R_{1}(\rho, c)=$ $\rho(1-\rho)(\rho-a)$ for some $0 \leq a \leq 1$, the global existence of solutions is proved in [2]. In the logistic-growth model $R_{1}(\rho, c)=\rho\left(1-\rho^{\gamma-1}\right)$, a global weak solution exists for all $\gamma>2-1 / d[29]$. These results have been obtained for the parabolic-elliptic model. 
Recently, a fourth way to obtain global existence of solutions has been proposed [14]. The idea is to add a cross-diffusion term in the equation for the chemical signal:

$$
\begin{aligned}
\partial_{t} \rho & =\operatorname{div}(\nabla \rho-\rho \nabla c), \\
\alpha \partial_{t} c & =\Delta c+\delta \Delta \rho+\rho-c \quad \text { in } \Omega, t>0,
\end{aligned}
$$

where $\delta>0$. At first sight, the additional cross-diffusion term seems to cause several mathematical difficulties since the diffusion matrix of the above system is neither symmetric nor positive definite, and we cannot apply the maximum principle to the equation for the chemical signal anymore. All these difficulties can be resolved by the observation that the above system possesses a logarithmic entropy,

$$
E_{0}(\rho, c)=\int_{\Omega}\left[\rho(\log \rho-1)+\alpha \frac{c^{2}}{2 \delta}\right] d x,
$$

allowing for global existence results and revealing some interesting structural properties of the system. In fact, the entropy production equation

$$
\frac{d E_{0}}{d t}+\int_{\Omega}\left(4|\nabla \sqrt{\rho}|^{2}+\frac{1}{\delta}|\nabla c|^{2}+\frac{1}{\delta} c^{2}\right) d x=\frac{1}{\delta} \int_{\Omega} \rho c d x
$$

and suitable Gagliardo-Nirenberg estimates for the right-hand side lead to gradient estimates for $\sqrt{\rho}$ and $c$. Another motivation for the introduction of the additional cross diffusion is that, whereas finite-element discretizations of the classical Keller-Segel model break down some time before the blow up, the numerical solutions to the augmented model exists for all time, which may lead to estimates of the blow-up time. This question is currently under investigation.

In [14], the existence of global weak solutions has been proved in the two-dimensional situation only. In this paper, we generalize this result to three space dimensions by allowing for nonlinearities in the cell diffusion terms. Since nonlinear diffusion in the cell equation helps to achieve global existence results (see above), we suggest, in contrast to [14], a nonlinear cross-diffusion term. More precisely, we consider the equations

$$
\begin{aligned}
\partial_{t} \rho & =\operatorname{div}\left(\nabla\left(\rho^{m}\right)-\rho \nabla c\right), \\
\alpha \partial_{t} c & =\Delta c+\delta \Delta\left(\rho^{n}\right)+\rho-c \quad \text { in } \Omega, t>0,
\end{aligned}
$$

subject to the no-flux and initial conditions

$$
\begin{aligned}
& \left(\nabla\left(\rho^{m}\right)-\rho \nabla c\right) \cdot \nu=\nabla\left(c+\delta \rho^{n}\right) \cdot \nu=0 \quad \text { on } \partial \Omega, t>0, \\
& \rho(\cdot, 0)=\rho_{0}, \alpha c(\cdot, 0)=\alpha c_{0} \quad \text { in } \Omega .
\end{aligned}
$$

Notice that these boundary conditions are equivalent to $\nabla \rho \cdot \nu=\nabla c \cdot \nu=0$ on $\partial \Omega$ for smooth positive solutions.

In two space dimensions, the case $m=n=1$ is covered by [14]. If $m>3-4 / d$, $2 \leq d \leq 3$, the nonlinear diffusion already prevents blow-up of the solutions without additional cross diffusion, see [20, 21, 22]. The question remains if we can allow for linear and fast diffusion of cells, $m \leq 1$, for some $n>1$, and still obtain global existence results. In this paper, we show that this is indeed true. For instance, we show that in the presence 
of the additional cross diffusion term and in three space dimensions, we can allow for the classical cell diffusion exponent $m=1$ and still obtain global existence results. This shows that the result of [14] can be generalized to the three-dimensional case if the cross diffusion is of degenerate type. These remarks motivate us to restrict ourselves to the case $m>0$ and $n>1$. Our first main result is as follows.

Theorem 1 (Global existence). Let $\Omega \subset \mathbb{R}^{d}(1 \leq d \leq 3)$ be a bounded domain with $\partial \Omega \in$ $C^{1,1}$. Let $\alpha \geq 0, m>0, n>1$, and let $p=(m+n-1) / 2$ satisfy $1-n / d<p \leq \min \{m, n\}$. Furthermore, let $0 \leq \rho_{0} \in L^{n}(\Omega)$ and $\alpha c_{0} \in L^{2}(\Omega)$. Then there exists a global weak solution $(\rho, c)$ to (1)-(4) satisfying $\rho \geq 0$ in $\Omega, t>0$, and, for some $s \in(1,2]$,

$$
\begin{aligned}
& \rho \in L_{\mathrm{loc}}^{\infty}\left(0, \infty ; L^{n}(\Omega)\right) \cap L_{\mathrm{loc}}^{2 Q}\left(0, \infty ; L^{2 Q}(\Omega)\right), \\
& \rho^{m}, \quad \rho^{n} \in L_{\mathrm{loc}}^{s}\left(0, \infty ; W^{1, s}(\Omega)\right), \quad \rho \nabla c \in L_{\mathrm{loc}}^{s}\left(0, \infty ; L^{s}(\Omega)\right), \\
& \alpha c \in L_{\mathrm{loc}}^{\infty}\left(0, \infty ; L^{2}(\Omega)\right), \quad c \in L_{\mathrm{loc}}^{2}\left(0, \infty ; H^{1}(\Omega)\right), \\
& \partial_{t} \rho, \quad \alpha \partial_{t} c \in L_{\mathrm{loc}}^{s}\left(0, \infty ;\left(W^{1, s}(\Omega)\right)^{\prime}\right),
\end{aligned}
$$

where $Q=n / d+p>1$.

Remark 2. A weak solution is to be understood in the standard sense by testing the system of equations against compactly supported smooth functions in $\left.C^{\infty}((0, T) \times \Omega)\right)$. Due to the regularity properties of the solution, however, test functions in $L^{s}\left(0, T ; W^{1, s}(\Omega)\right)$ are sufficient for the weak formulation of both equations in the fully parabolic system to be well defined. For the parabolic-elliptic system, we show in Section 4 that we can even allow for test functions in $L^{2}\left(0, T ; H^{1}(\Omega)\right)$.

Let us discuss the conditions on $p$ which are equivalent to

$$
m-1 \leq n \leq m+1, \quad m+n+\frac{2}{d} n>3 .
$$

The areas of admissible values for $(m, n)$ are illustrated in Figure 1. Notice that the bands between $n-1 \leq m \leq n+1$ continue to the right.
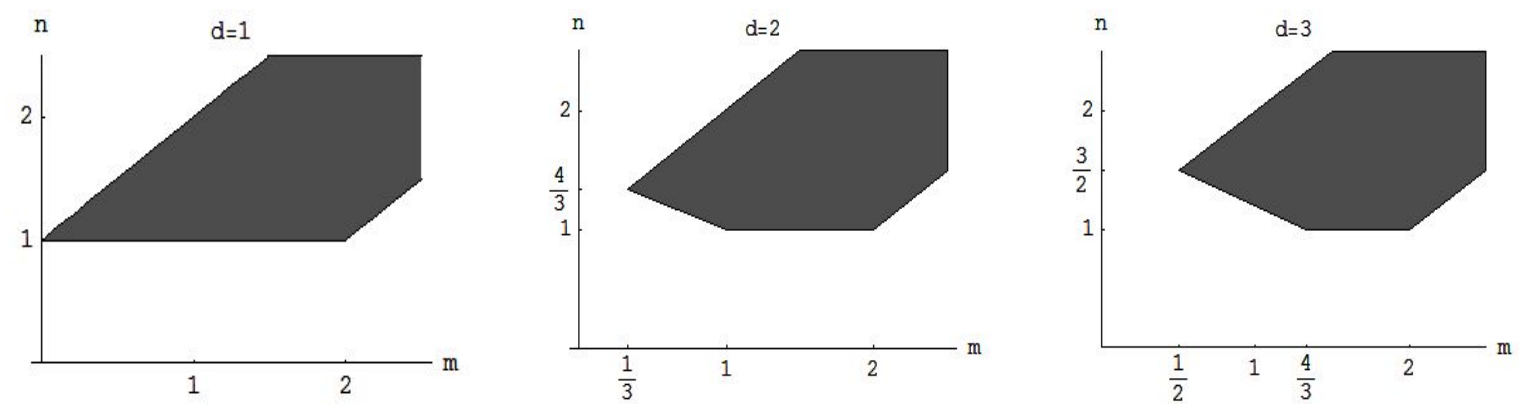

Figure 1. Admissible values $(m, n)$ for $d=1$ (left), $d=2$ (middle), and $d=3$ (right).

In the fast-diffusion case, for $d=2$, we may take $\frac{1}{3}<m<1$ and $\frac{1}{2}(3-m)<n \leq m+1$; for $d=3$, the values $\frac{1}{2}<m<1$ and $\frac{3}{5}(3-m)<n \leq m+1$ are admissible. For classical 
diffusion, $m=1$, the above conditions are satisfied for any $1<n \leq 2$ (if $d=2$ ) and $6 / 5<n \leq 2$ (if $d=3$ ). Hence, the degenerate cross-diffusion term prevents blow up in finite time even in the case of linear cell diffusion in three dimensions. In short, one of the conditions in (5) is needed to derive a nice bound on an entropy functional and the others for suitable compactness and continuity properties of the approximated sequences.

The key idea of the proof of Theorem 1 is the observation that system (1)-(2) possesses an entropy functional,

$$
E(\rho, c)=\int_{\Omega}\left(\frac{\rho^{n}}{n-1}+\alpha \frac{c^{2}}{2 \delta}\right) d x
$$

useful to derive a priori estimates. Indeed, differentiating formally this functional, we obtain the entropy production equation

$$
\frac{d E}{d t}+\int_{\Omega}\left(\frac{m n}{p^{2}}\left|\nabla \rho^{p}\right|^{2}+\frac{1}{\delta}|\nabla c|^{2}+\frac{c^{2}}{\delta}\right) d x=\frac{1}{\delta} \int_{\Omega} \rho c d x
$$

recalling that $p=(m+n-1) / 2$. We will show in the proof of Lemma 10 that the right-hand side can be estimated for any $\beta>0$ as follows:

$$
\int_{\Omega} \rho c d x \leq \beta \int_{\Omega}\left|\nabla \rho^{p}\right|^{2} d x+C\left(\beta,\|\rho\|_{L^{1}(\Omega)}\right)+\frac{1}{2} \int_{\Omega}\left(|\nabla c|^{2}+c^{2}\right) d x,
$$

under the restriction $1-2 / d<p$, which follows from the conditions in (5) for $1 \leq d \leq 3$. The assumptions $m>0$ and $n>1$ imply that $p>0$, thus only for $d=3$ we obtain the restriction $p>1 / 3$. Let us remark that in the case $d=3$, the restriction $1-2 / d<p$ is redundant, that is, conditions (5) together with $n>1$ and $m>0$ imply that $p+2 / d \geq$ $p+n / d>1$ for $n \in(1,2]$, as well as $p>1$ since $2 p=m+n-1>2$ for $n>2$ with $m \geq n-1 \geq 1$.

The existence of the entropy functional (6) implies the existence of so-called entropy variables which makes the new diffusion matrix positive (semi-) definite. Indeed, introducing the entropy variables

$$
r=\frac{\partial E}{\partial \rho}=\frac{n}{n-1} \rho^{n-1}, \quad b=\frac{\partial E}{\partial c}=\frac{c}{\delta},
$$

system (1)-(2) can be written as

$$
\frac{\partial}{\partial t}\left(\begin{array}{c}
\rho \\
\alpha c
\end{array}\right)-\operatorname{div}\left(\left(\begin{array}{cc}
(m / n) \rho^{m-n+1} & -\delta \rho \\
\delta \rho & \delta
\end{array}\right) \nabla\left(\begin{array}{l}
r \\
b
\end{array}\right)\right)=\left(\begin{array}{c}
0 \\
\rho-c
\end{array}\right) .
$$

In hyperbolic or parabolic systems, the existence of an entropy functional is equivalent to the existence of a change of unknowns which "symmetrizes" the system [8, 18]. (For parabolic systems, "symmetrization" means that the transformed diffusion matrix is symmetric and positive definite.) In system (8), the diffusion matrix is nonsymmetric, but still positive semi-definite.

The existence proof is based on the construction of a problem which approximates (8). First, we replace the time derivative by an implicit Euler approximation with time step $\tau>0$ and add a weak form of the fourth-order operator $\varepsilon\left(\Delta^{2} r-\operatorname{div}\left(|\nabla r|^{2} \nabla r\right)+r\right)(\varepsilon>0)$ to the first component of (8), which guarantees the coercivity of the elliptic system in 
$H^{2}(\Omega)$ with respect to $r$. The existence of weak approximating solutions $\left(r_{\varepsilon}, b_{\varepsilon}\right)$ is shown by using the Leray-Schauder fixed-point theorem. At this point, we need the restriction $p \leq m$, which is equivalent to $n \leq m+1$, in (5) to ensure the continuity and coercivity. The discrete entropy estimate implies a priori estimates uniform in the approximation parameters $\tau$ and $\varepsilon$, which allow us to pass to the limit $(\tau, \varepsilon) \rightarrow 0$.

There are two technical difficulties in the limiting procedure. The first one is that the entropy estimate yields a uniform bound for $\rho_{\varepsilon}^{p}$ in $H^{1}(\Omega)$, but an estimate for $\partial_{t} \rho_{\varepsilon}$ in $\left(H^{3}(\Omega)\right)^{\prime}$. If $p \leq 1$, this implies a bound for $\rho_{\varepsilon}$ in $W^{1, r}(\Omega)$ for some $r>0$, and we can apply the Aubin lemma to conclude the relative compactness of the family $\left(\rho_{\varepsilon}\right)_{\varepsilon>0}$. If $p>1$, we infer this property using a variant of the Dubinskii lemma (see Lemma 7 ). The second difficulty is to ensure the strong convergence of the family $\left(\rho_{\varepsilon}\right)_{\varepsilon>0}$ in $L^{2}(\Omega \times(0, T))$. In two space dimensions $d=2$ (and with $n=m=1$ ), this has been proved in [14]. However, for $d=3$ (and $p<1$ ), our uniform estimates in Lemma 12 need additional assumptions on the diffusion parameters, namely $p>1-n / d$ and $p \leq n$, or equivalently, the remaining two conditions in (5): $m+n+2 n / d>3$ and $m-1 \leq n$.

Our second main result concerns some qualitative properties of the solutions to (1)-(4) using the entropy functional. First, we prove $L^{\infty}$ bounds for the solutions to the parabolicelliptic system generalizing the results of $[21,22]$ to this situation.

Theorem 3 (Boundedness in $\left.L^{\infty}\right)$. Let the assumptions of Theorem 1 hold and let $\alpha=0$. Then, for any $T>0$, the solution $(\rho, c)$ to the parabolic-elliptic system (1)-(4) satisfies

$$
\|\rho\|_{L^{\infty}\left(0, T ; L^{\infty}(\Omega)\right)}+\|c\|_{L^{\infty}\left(0, T ; L^{\infty}(\Omega)\right)} \leq C(T),
$$

where the constant $C(T)>0$ depends on $T>0$.

Second, we are able to show the asymptotic stability of solutions to the constant steady state. Due to the special structure of the entropy functional, we can allow for a very particular choice of the parameters $m, n$, and $\delta$ only.

Proposition 4 (Long-time decay for $m=1, n=2)$. Let $\Omega \subset \mathbb{R}^{d}(1 \leq d \leq 3$ ) be a bounded domain with $\partial \Omega \in C^{1,1}$. Let $\rho_{0} \in L^{\infty}(\Omega), m=1, n=2$, and $\delta>C_{P}^{2} / 4$, where $C_{P}$ is the constant of the Poincare inequality in $L^{2}(\Omega)$. Then the solution to the parabolic-elliptic system (32)-(33) with $\alpha=0$, constructed in Theorem 15, decays exponentially fast to the homogeneous steady state in the sense that

$$
\left\|\rho(\cdot, t)-\rho^{*}\right\|_{L^{2}(\Omega)} \leq C e^{-\kappa t}, \quad\left\|c(\cdot, t)-c^{*}\right\|_{L^{1}(\Omega)} \leq C e^{-\kappa t},
$$

where $C>0$ is some constant and $\kappa=\min \left\{1,4 \delta-C_{P}^{2}\right\} /(4 \delta)$. Moreover, any smooth solution $(\rho, c)$ to the fully parabolic system (32)-(33) with $\alpha=1$ has the decay properties

$$
\left\|\rho(\cdot, t)-\rho^{*}\right\|_{L^{2}(\Omega)} \leq C e^{-\kappa t}, \quad\left\|c(\cdot, t)-c^{*}\right\|_{L^{2}(\Omega)} \leq C e^{-\kappa t}
$$

for all $t>0$.

The paper is organized as follows. In Section 2, we prove an inequality which is needed for the proof of (7) and we show a compactness result which combines the lemmas of Aubin and Dubinskii. Theorems 1 and 3 are shown in Sections 3 and 4, respectively, whereas 
Proposition 4 is proved in Section 5. Finally, in Section 6 we present some numerical results in two and three space dimensions which illustrate the effect of the exponent $n$.

\section{Auxiliary Results}

Lemma 5. Let $\Omega \subset \mathbb{R}^{d}(d \geq 1)$ be a bounded domain with $\partial \Omega \in C^{0,1}$. Furthermore, let $\beta>0$ and

- if $d \geq 3$ : either $1-2 / d<p \leq 1$ and $q=2 d /(d+2)$ or $p>1$ and $q=p+1 / 2$,

- if $d \leq 2$ : either $0<p \leq 1, q>1$, and $p+1 / q>3 / 2-1 / d$ or $p>1$ and $q=p+1 / 2$.

Then there exists a constant $C\left(\beta,\|\rho\|_{L^{1}(\Omega)}\right)>0$ such that for all $\rho \in L^{1}(\Omega)$ satisfying $\rho \geq 0$ in $\Omega$ and $\rho^{p} \in H^{1}(\Omega)$, the following inequality holds:

$$
\|\rho\|_{L^{q}(\Omega)}^{2} \leq \beta\left\|\nabla \rho^{p}\right\|_{L^{2}(\Omega)}^{2}+C\left(\beta,\|\rho\|_{L^{1}(\Omega)}\right) .
$$

Notice that the continuous embedding $H^{1}(\Omega) \hookrightarrow L^{s}(\Omega)$, where $1 \leq s \leq 2 d /(d-2)$ if $d \geq 3$ and $1 \leq s<\infty$ if $d \leq 2$, shows that $\rho^{p} \in H^{1}(\Omega)$ implies that $\rho \in L^{s p}(\Omega)$, and the condition $q \leq s p$ has to be imposed. This condition is satisfied for the above choices of $p$ and $q$.

Proof. First, let $0<p \leq 1$ and $(p, q)$ be given as in the Lemma. The Gagliardo-Nirenberg inequality, see e.g. [10, Theorem 10.1] and [31, Theorem 1.1.4], gives

$$
\begin{aligned}
\|\rho\|_{L^{q}(\Omega)}^{2} & =\left\|\rho^{p}\right\|_{L^{q / p}(\Omega)}^{2 / p} \leq C\left\|\nabla \rho^{p}\right\|_{L^{2}(\Omega)}^{2 \theta / p}\left\|\rho^{p}\right\|_{L^{1 / p}(\Omega)}^{2(1-\theta) / p}+C\left\|\rho^{p}\right\|_{L^{1 / p}(\Omega)}^{2 / p} \\
& =C\left\|\nabla \rho^{p}\right\|_{L^{2}(\Omega)}^{2 \theta / p}\|\rho\|_{L^{1}(\Omega)}^{2(1-\theta)}+C\|\rho\|_{L^{1}(\Omega)}^{2},
\end{aligned}
$$

where $\theta=d p(1-1 / q) /(1-d / 2+d p)$ and $C>0$ is here and in the following a generic constant. The conditions $p>1-2 / d$ if $d \geq 3$ and $q>1$ if $d \leq 2$ imply that $\theta>0$. For all space dimensions, it holds that $p+1 / q>3 / 2-1 / d$ which is equivalent to $\theta<p \leq 1$. Then the inequality $\theta / p<1$ allows us to apply the Young inequality:

$$
\|\rho\|_{L^{q}(\Omega)}^{2} \leq \beta\left\|\nabla \rho^{p}\right\|_{L^{2}(\Omega)}^{2}+C(\beta)\|\rho\|_{L^{1}(\Omega)}^{2 p(1-\theta) /(p-\theta)}+C\|\rho\|_{L^{1}(\Omega)}^{2},
$$

proving the first case.

Next, let $p>1$ and $q=p+1 / 2$. Notice that the Poincaré inequality implies that

$$
\|f\|_{L^{2}(\Omega)} \leq\left\|f-\int_{\Omega} f d x\right\|_{L^{2}(\Omega)}+\|f\|_{L^{1}(\Omega)} \leq C_{P}\|\nabla f\|_{L^{2}(\Omega)}+\|f\|_{L^{1}(\Omega)} .
$$

This together with the Hölder inequality leads to

$$
\begin{aligned}
\|\rho\|_{L^{q}(\Omega)}^{2} & =\left\|\rho^{q}\right\|_{L^{1}(\Omega)}^{2 / q}=\left\|\rho^{p} \rho^{1 / 2}\right\|_{L^{1}(\Omega)}^{2 / q} \\
& \leq\left\|\rho^{p}\right\|_{L^{2}(\Omega)}^{2 / q}\left\|\rho^{1 / 2}\right\|_{L^{2}(\Omega)}^{2 / q} \leq C\left(\left\|\nabla \rho^{p}\right\|_{L^{2}(\Omega)}^{2 / q}+\left\|\rho^{p}\right\|_{L^{1}(\Omega)}^{2 / q}\right)\left\|\rho^{1 / 2}\right\|_{L^{2}(\Omega)}^{2 / q} \\
& =C\left\|\nabla \rho^{p}\right\|_{L^{2}(\Omega)}^{2 / q}\|\rho\|_{L^{1}(\Omega)}^{1 / q}+C\|\rho\|_{L^{p}(\Omega)}^{2 p / q}\|\rho\|_{L^{1}(\Omega)}^{1 / q} .
\end{aligned}
$$


Furthermore, using the interpolation inequality with $1 / p=\theta / q+(1-\theta) / 1$ or, equivalently, $p \theta / q=(p-1) /(q-1)>0$,

$$
\|\rho\|_{L^{q}(\Omega)}^{2} \leq C\left\|\nabla \rho^{p}\right\|_{L^{2}(\Omega)}^{2 / q}\|\rho\|_{L^{1}(\Omega)}^{1 / q}+C\|\rho\|_{L^{q}(\Omega)}^{2 p \theta / q}\|\rho\|_{L^{1}(\Omega)}^{2 p(1-\theta) / q}\|\rho\|_{L^{1}(\Omega)}^{1 / q} .
$$

Since $q>1$, we may employ the Young inequality for the first summand to obtain

$$
\|\rho\|_{L^{q}(\Omega)}^{2} \leq \beta\left\|\nabla \rho^{p}\right\|_{L^{2}(\Omega)}^{2}+C(\beta)\|\rho\|_{L^{1}(\Omega)}^{1 /(q-1)}+C\|\rho\|_{L^{q}(\Omega)}^{2 p \theta / q}\|\rho\|_{L^{1}(\Omega)}^{2 p(1-\theta) / q+1 / q} .
$$

Since $1<p<q$, it follows that $2 p \theta / q<2$, which allows us to use the Young inequality for the second summand:

$$
\|\rho\|_{L^{q}(\Omega)}^{2} \leq \beta\left\|\nabla \rho^{p}\right\|_{L^{2}(\Omega)}+C(\beta)\|\rho\|_{L^{1}(\Omega)}^{1 /(q-1)}+\frac{1}{2}\|\rho\|_{L^{q}(\Omega)}^{2}+C\left(\beta,\|\rho\|_{L^{1}(\Omega)}\right) .
$$

The lemma is proved.

Next, we recall a compactness result. Let $\left(\sigma_{h} \rho\right)(x, t)=\rho(x, t-h), t \geq h>0$, be a shift operator.

Lemma 6 (Dubinskii). Let $\Omega \subset \mathbb{R}^{d}(d \geq 1)$ be a bounded domain with $\partial \Omega \in C^{0,1}$ and let $T>0$. Furthermore, let $p \geq 1, q \geq 1$, and $s \geq 0$, and let $\left(\rho_{\varepsilon}\right)$ be a sequence of nonnegative functions satisfying

$$
h^{-1}\left\|\rho_{\varepsilon}-\sigma_{h} \rho_{\varepsilon}\right\|_{L^{1}\left(h, T ;\left(H^{s}(\Omega)\right)^{\prime}\right)}+\left\|\rho_{\varepsilon}^{p}\right\|_{L^{q}\left(0, T ; H^{1}(\Omega)\right)} \leq C \quad \text { for all } h>0,
$$

where $C>0$ is a constant which is independent of $\varepsilon$ and $h$. Then $\left(\rho_{\varepsilon}\right)$ is relatively compact in $L^{p \ell}\left(0, T ; L^{p r}(\Omega)\right)$ for all $\ell<q$ and for all $r<2 d /(d-2)$ if $d \geq 3, r<\infty$ if $d=2$, and $r \leq \infty$ if $d=1$.

A variant of this lemma is due to Dubinskii, see [23, Théorème 21.1, Chapter 1] for a proof. A simple proof is achieved by applying the lemmas of Aubin [27] and Chavent-Jaffre [5]. Since the result is of interest by itself, we provide the (short) proof.

Proof. The function $f(x)=x^{1 / p}, 0<x<\infty$, is Hölder continuous with exponent $1 / p$. Therefore, with $u=\rho_{\varepsilon}^{p}$, by the lemma of Chavent-Jaffre [5, p. 141],

$$
\left\|\rho_{\varepsilon}\right\|_{W^{1 / p, 2 p}(\Omega)}=\|f(u)\|_{W^{1 / p, 2 p}(\Omega)} \leq K\|u\|_{H^{1}(\Omega)}^{1 / p}=C\left\|\rho_{\varepsilon}^{p}\right\|_{H^{1}(\Omega)}^{1 / p} .
$$

This shows that $\left(\rho_{\varepsilon}\right)$ is bounded in $L^{p q}\left(0, T ; W^{1 / p, 2 p}(\Omega)\right)$. By Aubin's lemma [27, Theorem $6]$ and the compact embedding $W^{1 / p, 2 p}(\Omega) \hookrightarrow L^{p r}(\Omega)$ ( $r$ is as in the lemma), $\left(\rho_{\varepsilon}\right)$ is relatively compact in $L^{p \ell}\left(0, T ; L^{p r}(\Omega)\right)$ for all $\ell<q$.

The following result, which will be used in this paper, is a consequence of Lemma 6 .

Lemma 7. Let $\Omega \subset \mathbb{R}^{d}(d \geq 1)$ be a bounded domain with $\partial \Omega \in C^{0,1}$, let $T>0, \tau>0$, and let $t_{k}=k \tau, k=0, \ldots, N$, with $N \tau=T$ be a decomposition of the interval $[0, T]$. Furthermore, let $p \geq 1, q \geq 1$, and $s \geq 0$, and let $\left(\rho_{\tau}\right)$ be a sequence of nonnegative functions, which are piecewise constant in time on $(0, T)$, satisfying

$$
\tau^{-1}\left\|\rho_{\tau}-\sigma_{\tau} \rho_{\tau}\right\|_{L^{1}\left(\tau, T ;\left(H^{s}(\Omega)\right)^{\prime}\right)}+\left\|\rho_{\tau}^{p}\right\|_{L^{q}\left(0, T ; H^{1}(\Omega)\right)} \leq C \quad \text { for all } \tau>0,
$$


where $C>0$ is a constant which is independent of $\tau$. Then $\left(\rho_{\tau}\right)$ is relatively compact in $L^{p \ell}\left(0, T ; L^{p r}(\Omega)\right)$ for all $\ell<q$ and for all $r<2 d /(d-2)$ if $d \geq 3, r<\infty$ if $d=2$, and $r \leq \infty$ if $d=1$.

Proof. Since $\rho_{\tau}$ is piecewise constant in time, we can write $\rho_{\tau}(\cdot, t)=\rho_{k}$ for $t \in((k-1) \tau, k \tau]$, $k=1, \ldots, N$, for some functions $\rho_{k}$.

Case $h<\tau$. The difference $\rho_{\tau}-\sigma_{h} \rho_{\tau}$ partially cancels for $h<\tau$, and we obtain, for $k=1, \ldots, N-1$ and $t>h$,

$$
\left\|\rho_{\tau}(\cdot, t)-\left(\sigma_{h} \rho_{\tau}\right)(\cdot, t)\right\|_{\left(H^{s}(\Omega)\right)^{\prime}}= \begin{cases}\left\|\rho_{k+1}-\rho_{k}\right\|_{\left(H^{s}(\Omega)\right)^{\prime}} & \text { if } t_{k}<t \leq t_{k}+h, \\ 0 & \text { else. }\end{cases}
$$

Therefore, by assumption,

$$
\begin{aligned}
h^{-1}\left\|\rho_{\tau}-\sigma_{h} \rho_{\tau}\right\|_{L^{1}\left(h, T ;\left(H^{s}(\Omega)\right)^{\prime}\right)} & =h^{-1} \sum_{k=1}^{N-1} \int_{t_{k}}^{t_{k}+h}\left\|\rho_{k+1}-\rho_{k}\right\|_{\left(H^{s}(\Omega)\right)^{\prime}} d t \\
= & \sum_{k=1}^{N-1}\left\|\rho_{k+1}-\rho_{k}\right\|_{\left(H^{s}(\Omega)\right)^{\prime}}=\tau^{-1} \sum_{k=1}^{N-1} \int_{t_{k}}^{t_{k+1}}\left\|\rho_{\tau}(\cdot, t)-\left(\sigma_{\tau} \rho_{\tau}\right)(\cdot, t)\right\|_{\left(H^{s}(\Omega)\right)^{\prime}} d t \leq C
\end{aligned}
$$

uniformly in $h<\tau$.

Case $h \geq \tau$. There exists $m \in \mathbb{N}$ such that $t_{m}<h \leq t_{m+1}$. Then, for $t \in\left(t_{k+m-1}, t_{k+m}\right]$, $k=1, \ldots N-m$,

$$
\left\|\left(\rho_{\tau}-\sigma_{h} \rho_{\tau}\right)(\cdot, t)\right\|_{\left(H^{s}(\Omega)\right)^{\prime}}= \begin{cases}\left\|\rho_{k+m}-\rho_{k-1}\right\|_{\left(H^{s}(\Omega)\right)^{\prime}} & \text { if } t_{k+m-1}<t \leq t_{k-1}+h \\ \left\|\rho_{k+m}-\rho_{k}\right\|_{\left(H^{s}(\Omega)\right)^{\prime}} & \text { if } t_{k-1}+h<t \leq t_{k+m}\end{cases}
$$

We compute

$$
\begin{aligned}
\| \rho_{\tau}- & \sigma_{h} \rho_{\tau} \|_{L^{1}\left(h, T ;\left(H^{s}(\Omega)\right)^{\prime}\right)} \\
= & \int_{h}^{t_{m+1}}\left\|\rho_{\tau}-\sigma_{h} \rho_{\tau}\right\|_{\left(H^{s}(\Omega)\right)^{\prime}} d t+\sum_{k=2}^{N-m} \int_{t_{k+m-1}}^{t_{k+m}}\left\|\rho_{\tau}-\sigma_{h} \rho_{\tau}\right\|_{\left(H^{s}(\Omega)\right)^{\prime}} d t \\
= & \int_{h}^{t_{m+1}}\left\|\rho_{m+1}-\rho_{1}\right\|_{\left(H^{s}(\Omega)\right)^{\prime}} d t+\sum_{k=2}^{N-m} \int_{t_{k+m-1}}^{t_{k-1}+h}\left\|\rho_{k+m}-\rho_{k-1}\right\|_{\left(H^{s}(\Omega)\right)^{\prime}} d t \\
& +\sum_{k=2}^{N-m} \int_{t_{k-1}+h}^{t_{k+m}}\left\|\rho_{k+m}-\rho_{k}\right\|_{\left(H^{s}(\Omega)\right)^{\prime}} d t \\
= & \left(t_{m+1}-h\right)\left\|\rho_{m+1}-\rho_{1}\right\|_{\left(H^{s}(\Omega)\right)^{\prime}}+\left(h-t_{m}\right) \sum_{k=2}^{N-m}\left\|\rho_{k+m}-\rho_{k-1}\right\|_{\left(H^{s}(\Omega)\right)^{\prime}} \\
& +\left(t_{m+1}-h\right) \sum_{k=2}^{N-m}\left\|\rho_{k+m}-\rho_{k}\right\|_{\left(H^{s}(\Omega)\right)^{\prime} .}
\end{aligned}
$$


We employ the estimates $h-t_{m} \leq \tau, t_{m+1}-h \leq \tau$ and the triangle inequality:

$$
\begin{aligned}
& \left\|\rho_{\tau}-\sigma_{h} \rho_{\tau}\right\|_{L^{1}\left(h, T ;\left(H^{s}(\Omega)\right)^{\prime}\right)} \leq \tau \sum_{j=2}^{m+1}\left\|\rho_{j}-\rho_{j-1}\right\|_{\left(H^{s}(\Omega)\right)^{\prime}} \\
& \quad+\tau \sum_{j=0}^{m} \sum_{k=2}^{N-m}\left\|\rho_{k+j}-\rho_{k+j-1}\right\|_{\left(H^{s}(\Omega)\right)^{\prime}}+\tau \sum_{j=0}^{m} \sum_{k=2}^{N-m}\left\|\rho_{k+j}-\rho_{k+j-1}\right\|_{\left(H^{s}(\Omega)\right)^{\prime}} .
\end{aligned}
$$

Since

$$
\sum_{k=2}^{N-m} \sum_{j=i}^{m} a_{k+j}=\sum_{j=i}^{m} \sum_{\ell=2+j}^{N-m+j} a_{\ell} \leq(m-i+1) \sum_{\ell=2}^{N} a_{\ell}
$$

for numbers $a_{\ell} \geq 0$ and all $0 \leq i \leq m$, it follows that

$$
\begin{aligned}
\left\|\rho_{\tau}-\sigma_{h} \rho_{\tau}\right\|_{L^{1}\left(h, T ;\left(H^{s}(\Omega)\right)^{\prime}\right)} & \leq 3(m+1) \tau \sum_{k=1}^{N-1}\left\|\rho_{k+1}-\rho_{k}\right\|_{\left(H^{s}(\Omega)\right)^{\prime}} \\
& =3(m+1) \int_{\tau}^{T}\left\|\rho_{\tau}-\sigma_{\tau} \rho_{\tau}\right\|_{\left(H^{s}(\Omega)\right)^{\prime}} d t .
\end{aligned}
$$

Thus, using $(m+1) \tau \leq h+\tau \leq 2 h$,

$$
\left\|\rho_{\tau}-\sigma_{h} \rho_{\tau}\right\|_{L^{1}\left(h, T ;\left(H^{s}(\Omega)\right)^{\prime}\right)} \leq \frac{6 h}{\tau}\left\|\rho_{\tau}-\sigma_{\tau} \rho_{\tau}\right\|_{L^{1}\left(\tau, T ;\left(H^{s}(\Omega)\right)^{\prime}\right)} \leq C h .
$$

We conclude that in both cases, for all $h>0$,

$$
\left\|\rho_{\tau}-\sigma_{h} \rho_{\tau}\right\|_{L^{1}\left(h, T ;\left(H^{s}(\Omega)\right)^{\prime}\right)} \leq C h,
$$

and this estimate is uniform in $\tau>0$. We apply Lemma 6 to conclude the result.

\section{Global existence of Weak solutions}

In this section, we prove Theorem 1. Let in the following $\Omega \subset \mathbb{R}^{d}(1 \leq d \leq 3)$ be a bounded domain with $\partial \Omega \in C^{1,1}$. The smoothness assumption on the boundary of the domain is needed for applying elliptic regularity results.

3.1. Solution of an approximate problem. We show first the existence of a weak solution to an approximate problem which is obtained by semi-discretizing (1)-(2) with respect to time and by regularizing the equation for the cell density. For this, let $T>0$ and $K \in \mathbb{N}$ and split the time interval in the subintervals

$$
(0, T]=\bigcup_{k=1}^{K}((k-1) \tau, k \tau], \quad \tau=T / K .
$$


For given $\left(\rho_{k-1}, c_{k-1}\right)$, which approximates $(\rho, c)$ at time $\tau(k-1)$, we wish to solve the approximate problem in the weak formulation

$$
\begin{aligned}
& \frac{1}{\tau} \int_{\Omega}\left(\left(\rho_{k}-\rho_{k-1}\right) \phi+\alpha\left(c_{k}-c_{k-1}\right) \psi\right) d x \\
& \quad+\int_{\Omega}\left(\begin{array}{c}
\nabla \phi \\
\nabla \psi
\end{array}\right)^{\top}\left(\begin{array}{cc}
(m / n) \rho_{k}^{m-n+1} & -\delta \rho_{k} \\
\delta \rho_{k} & \delta
\end{array}\right)\left(\begin{array}{c}
\nabla r_{k} \\
\nabla b_{k}
\end{array}\right) d x \\
& \quad+\varepsilon \int_{\Omega}\left(\Delta r_{k} \Delta \phi+\left|\nabla r_{k}\right|^{2} \nabla r_{k} \cdot \nabla \phi+r_{k} \phi\right) d x=\int_{\Omega}\left(\rho_{k}-c_{k}\right) \psi d x
\end{aligned}
$$

where the entropy variables are given by

$$
r_{k}=\frac{n}{n-1} \rho_{k}^{n-1}, \quad b_{k}=\frac{c_{k}}{\delta},
$$

and $(\phi, \psi) \in H^{2}(\Omega) \times H^{1}(\Omega)$ is a test function pair, well defined for $n>1$. We prove now the existence of a solution to (9) recalling that

$$
p=\frac{m+n-1}{2}
$$

Remark 8. For proving the existence of weak solutions, the regularization $\varepsilon \int_{\Omega}\left(\Delta r_{k} \Delta \phi+\right.$ $\left.r_{k} \phi\right) d x$ would be sufficient. The additional term is helpful when deriving energy estimates which lead to the uniform boundedness of the solutions to the parabolic-elliptic system, see Section 4.

Proposition 9. Let $\Omega \subset \mathbb{R}^{d}(1 \leq d \leq 3)$ be a bounded domain with $\partial \Omega \in C^{1,1}$. Furthermore, let $\left(r_{k-1}, b_{k-1}\right) \in L^{n /(n-1)}(\Omega) \times L^{2}(\Omega)$ with $r_{k-1} \geq 0$ in $\Omega$ and let $m>0, n>1$ be such that $1-2 / d<p \leq m$ with $p$ given by (10). Then there exists a weak solution $\left(r_{k}, b_{k}\right) \in H^{2}(\Omega) \times H^{1}(\Omega)$ to $(9)$ satisfying $r_{k} \geq 0$ in $\Omega$.

Proof. Step 1: Formulation of a modified problem. In order to solve $(9)$ in terms of $(r, b)$, we set

$$
w(r)=\rho=\left(\frac{n-1}{n} r\right)^{1 /(n-1)}, \quad r_{+}=\max \{0, r\} .
$$

We wish to solve first the system

$$
\begin{aligned}
& \frac{1}{\tau} \int_{\Omega}\left(\left(w\left(r_{+}\right)-w\left(r_{k-1}\right)\right) \phi+\alpha \delta\left(b_{k}-b_{k-1}\right) \psi\right) d x \\
& \quad+\int_{\Omega}\left(\begin{array}{c}
\nabla \phi \\
\nabla \psi
\end{array}\right)^{\top}\left(\begin{array}{cc}
(m / n) w\left(r_{+}\right)^{m-n+1} & -\delta w\left(r_{+}\right) \\
\delta w\left(r_{+}\right) & \delta
\end{array}\right)\left(\begin{array}{c}
\nabla r \\
\nabla b
\end{array}\right) d x \\
& \quad+\varepsilon \int_{\Omega}\left(\Delta r \Delta \phi+|\nabla r|^{2} \nabla r \cdot \nabla \phi+r \phi\right) d x=\int_{\Omega}\left(w\left(r_{+}\right)-\delta b\right) \psi d x
\end{aligned}
$$

where $(\phi, \psi) \in H^{2}(\Omega) \times H^{1}(\Omega)$. Notice that the assumption $p \leq m$ is equivalent to $m-n+1 \geq 0$, which is needed for the term $w\left(r_{+}\right)^{m-n+1}$ to be well defined. The minimum principle shows that any weak solution $(r, b)$ to this problem satisfies $r \geq 0$ in $\Omega$. Indeed, 
using $\left(r_{-}, 0\right)$, where $r_{-}=\min \{0, r\}$, as a test function, and observing that $w\left(r_{+}\right) \nabla r_{-}=0$, we obtain

$$
-\frac{1}{\tau} \int_{\Omega} w\left(r_{k-1}\right) r_{-} d x+\frac{m}{n} \int_{\Omega} w\left(r_{+}\right)^{m-n+1}\left|\nabla r_{-}\right|^{2} d x+\varepsilon \int_{\Omega}\left(\left(\Delta r_{-}\right)^{2}+\left|\nabla r_{-}\right|^{4}+r_{-}^{2}\right) d x=0 .
$$

Since all three integrals on the right-hand side are nonnegative, we conclude that $r_{-}=0$ and $r \geq 0$ in $\Omega$.

Step 2: The linearized problem. Let $\sigma \in[0,1]$ and $(\bar{r}, \bar{b}) \in H^{7 / 4}(\Omega) \times L^{2}(\Omega)$ be given. The Sobolev embedding $H^{7 / 4}(\Omega) \hookrightarrow C^{0}(\bar{\Omega})$ for $d \leq 3$ shows that $w\left(\bar{r}_{+}\right)$is bounded. Hence, the following linear problem is well defined:

$$
a((r, b),(\phi, \psi))=\sigma f(\phi, \psi) \quad \text { for all }(\phi, \psi) \in H^{2}(\Omega) \times H^{1}(\Omega),
$$

where

$$
\begin{aligned}
a((r, b),(\phi, \psi))= & \int_{\Omega}\left(\begin{array}{c}
\nabla \phi \\
\nabla \psi
\end{array}\right)^{\top}\left(\begin{array}{cc}
(m / n) w\left(\bar{r}_{+}\right)^{m-n+1} & -\delta w\left(\bar{r}_{+}\right) \\
\delta w\left(\bar{r}_{+}\right) & \delta
\end{array}\right)\left(\begin{array}{c}
\nabla r \\
\nabla b
\end{array}\right) d x \\
& +\varepsilon \int_{\Omega}\left(\Delta r \Delta \phi+|\nabla \bar{r}|^{2} \nabla r \cdot \nabla \phi+r \phi\right) d x+\delta \int_{\Omega} b \psi d x \\
f(\phi, \psi)= & -\frac{1}{\tau} \int_{\Omega}\left(\left(w\left(\bar{r}_{+}\right)-w\left(r_{k-1}\right)\right) \phi+\alpha \delta\left(\bar{b}-b_{k-1}\right) \psi\right) d x+\int_{\Omega} w\left(\bar{r}_{+}\right) \psi d x .
\end{aligned}
$$

The function $a:\left(H^{2}(\Omega) \times H^{1}(\Omega)\right)^{2} \rightarrow \mathbb{R}$ is bilinear and continuous due to the Sobolev embedding $H^{2}(\Omega) \subset H^{7 / 4}(\Omega) \subset W^{1,4}(\Omega) \subset C^{0}(\bar{\Omega})$ for $d \leq 3$. Here, we need the assumption $m-n+1 \geq 0$. The function $f: H^{2}(\Omega) \times H^{1}(\Omega) \rightarrow \mathbb{R}$ is linear and bounded which is a consequence of the estimate

$$
\int_{\Omega} w\left(r_{k-1}\right) \phi d x \leq\left\|w\left(r_{k-1}\right)\right\|_{L^{n}(\Omega)}\|\phi\|_{L^{n /(n-1)}(\Omega)} \leq C\|\phi\|_{H^{2}(\Omega)},
$$

for some constant $C>0$, since $r_{k-1} \in L^{n /(n-1)}(\Omega)$ gives $w\left(r_{k-1}\right) \in L^{n}(\Omega)$. Moreover, $a$ is coercive:

$$
\begin{aligned}
a((r, b),(r, b))= & \int_{\Omega}\left(\frac{m}{n} w\left(\bar{r}_{+}\right)^{m-n+1}|\nabla r|^{2}+\delta|\nabla b|^{2}\right) d x \\
& +\varepsilon \int_{\Omega}\left((\Delta r)^{2}+|\nabla \bar{r}|^{2}|\nabla r|^{2}+r^{2}\right) d x+\delta \int_{\Omega} b^{2} d x \\
\geq & C(\varepsilon, \delta)\left(\|r\|_{H^{2}(\Omega)}^{2}+\|b\|_{H^{1}(\Omega)}^{2}\right),
\end{aligned}
$$

for some constant $C(\varepsilon, \delta)>0$, since $\partial \Omega \in C^{1,1}$ (see Troianiello [28], p. 194). The LaxMilgram lemma now implies the existence and uniqueness of a solution $(r, b) \in H^{2}(\Omega) \times$ $H^{1}(\Omega)$ to $(12)$.

Step 3: The nonlinear problem. The previous step allows us to define the fixed-point operator $S:[0,1] \times H^{7 / 4}(\Omega) \times L^{2}(\Omega) \rightarrow H^{7 / 4}(\Omega) \times L^{2}(\Omega)$ by $S(\sigma, \bar{r}, \bar{b})=(r, b)$, where $(r, b) \in H^{2}(\Omega) \times H^{1}(\Omega)$ is the unique solution to (12). It holds $S(0, \bar{r}, \bar{b})=(0,0)$ for all $(\bar{r}, \bar{b}) \in H^{7 / 4}(\Omega) \times L^{2}(\Omega)$. Standard arguments prove that $S$ is continuous and compact, taken into account the compact embedding of $H^{2}(\Omega) \times H^{1}(\Omega)$ into $H^{7 / 4}(\Omega) \times L^{2}(\Omega)$. 
It remains to show that there exists a constant $C>0$ such that for all fixed points $(r, b) \in H^{7 / 4}(\Omega) \times L^{2}(\Omega)$ and $\sigma \in[0,1]$ satisfying $S(\sigma, r, b)=(r, b)$, the estimate

$$
\|(r, b)\|_{H^{7 / 4}(\Omega) \times L^{2}(\Omega)} \leq C
$$

holds. Let $(r, b)$ be such a fixed point. Let us first assume that $\sigma=1$. Then $(r, b)$ is a solution to (9). By the first step of the proof, we have $r \geq 0$ in $\Omega$. Moreover, we can easily derive a uniform $L^{1}$ bound for $\rho=w(r)$ by employing $(1,0)$ as a test function in $(9)$ :

$$
\int_{\Omega} \rho d x=\int_{\Omega} \rho_{k-1} d x-\tau \varepsilon \int_{\Omega} r d x \leq \int_{\Omega} \rho_{k-1} d x
$$

since $r$ is nonnegative. By iteration, we infer that

$$
\|\rho\|_{L^{1}(\Omega)} \leq\left\|\rho_{0}\right\|_{L^{1}(\Omega)} .
$$

The uniform estimate (13) is a consequence of the following discrete entropy estimate, which settles the case $\sigma=1$. The case $\sigma<1$ can be treated similarly.

Lemma 10. Let $(r, b) \in H^{2}(\Omega) \times H^{1}(\Omega)$ be a solution to (9) and let $1-2 / d<p \leq m$. Then

$$
\begin{aligned}
E(\rho, c) & +\frac{\tau m n}{2 p^{2}}\left\|\nabla \rho^{p}\right\|_{L^{2}(\Omega)}^{2}+\frac{\tau}{2 \delta}\|c\|_{H^{1}(\Omega)}^{2} \\
& +\varepsilon \tau\left(\|\Delta r\|_{L^{2}(\Omega)}^{2}+\|\nabla r\|_{L^{4}(\Omega)}^{4}+\|r\|_{L^{2}(\Omega)}^{2}\right) \leq E\left(\rho_{k-1}, c_{k-1}\right)
\end{aligned}
$$

where the entropy $E(\rho, c)$ is defined in $(6), \rho=w(r)=((n-1) r / n)^{1 /(n-1)}, c=\delta b$, and $p$ is defined in (10).

In order to prove this lemma, we employ the test function $(r, b)=\left(n \rho^{n-1} /(n-1), c / \delta\right)$ in (9):

$$
\begin{aligned}
& \frac{1}{\tau} \int_{\Omega}\left(\frac{n}{n-1} \rho^{n-1}\left(\rho-\rho_{k-1}\right)+\frac{\alpha}{\delta} c\left(c-c_{k-1}\right)\right) d x \\
& \quad+\int_{\Omega}\left(\frac{m n}{p^{2}}\left|\nabla \rho^{p}\right|^{2}+\frac{1}{\delta}\left(|\nabla c|^{2}+c^{2}\right)\right) d x+\varepsilon \int_{\Omega}\left((\Delta r)^{2}+|\nabla r|^{4}+r^{2}\right) d x=\frac{1}{\delta} \int_{\Omega} \rho c d x
\end{aligned}
$$

where $\rho_{k-1}=w\left(r_{k-1}\right)$ and $c_{k-1}=\delta b_{k-1}$. Since $n>1$, the mapping $g(x)=x^{n}, x \geq 0$, is convex, which implies the inequality $g(x)-g(y) \leq g^{\prime}(x)(x-y)$ for all $x, y \geq 0$. Hence, the first integral on the left-hand side of (15) is bounded from below by

$$
\frac{1}{\tau} \int_{\Omega}\left(\frac{1}{n-1}\left(\rho^{n}-\rho_{k-1}^{n}\right)+\frac{\alpha}{2 \delta}\left(c^{2}-c_{k-1}^{2}\right)\right) d x=\frac{1}{\tau}\left(E(\rho, c)-E\left(\rho_{k-1}, c_{k-1}\right)\right) .
$$

For the estimate of the right-hand side of (15), we employ first the Hölder and Young inequalities:

$$
\frac{1}{\delta} \int_{\Omega} \rho c d x \leq \frac{1}{\delta}\|\rho\|_{L^{q}(\Omega)}\|c\|_{L^{q^{\prime}}(\Omega)} \leq \frac{1}{2 \delta}\|\rho\|_{L^{q}(\Omega)}^{2}+\frac{1}{2 \delta}\|c\|_{H^{1}(\Omega)}^{2}
$$


where $q \geq 6 / 5$ if $d=3,1<q<\infty$ if $d \leq 2$, and $q^{\prime}=q /(q-1)$. In the last step, we have used the continuous embedding $H^{1}(\Omega) \hookrightarrow L^{q^{\prime}}(\Omega)$ which is valid since $q^{\prime} \leq 6$ if $d=3$ and $q^{\prime}<\infty$ if $d \leq 2$. By Lemma 5, we find that

$$
\frac{1}{\delta} \int_{\Omega} \rho c d x \leq \frac{m n}{2 p^{2}}\left\|\nabla \rho^{p}\right\|_{L^{2}(\Omega)}^{2}+C\left(\delta,\|\rho\|_{L^{1}(\Omega)}\right)+\frac{1}{2 \delta}\|c\|_{H^{1}(\Omega)}^{2} .
$$

The assumptions of the lemma are clearly satisfied for $d \leq 2$. If $d=3$ we can choose $q=2 d /(d+2)=6 / 5$ for $p \leq 1$ and $q=p+1 / 2>6 / 5$ for $p>1$. Putting together the above estimates and the $L^{1}$ bound (14), this finishes the proof of Lemma 10 and of Proposition 9 .

3.2. Uniform estimates. Let $\left(r_{k}, b_{k}\right)$ be a solution to the approximated problem (9) and set $\rho_{k}=w\left(r_{k}\right), c_{k}=\delta b_{k}$. We define the piecewise constant functions

$$
\left(\rho^{(\varepsilon, \tau)}, r^{(\varepsilon, \tau)}, c^{(\varepsilon, \tau)}\right)(x, t)=\left(\rho_{k}, r_{k}, c_{k}\right)(x) \quad \text { for } x \in \Omega, t \in((k-1) \tau, k \tau] .
$$

We denote by $D_{\tau} \rho(\cdot, t)=(\rho(\cdot, t)-\rho(\cdot, t-\tau)) / \tau$ the discrete time derivative of $\rho(\cdot, t)$, where $t \geq \tau$. In terms of the variables $\left(\rho^{(\varepsilon, \tau)}, c^{(\varepsilon, \tau)}\right)$, system (11) can be formulated as

$$
\begin{aligned}
0= & \int_{\tau}^{T}\left\langle D_{\tau} \rho^{(\varepsilon, \tau)}, \phi\right\rangle d t+\int_{\tau}^{T} \int_{\Omega}\left(\nabla\left(\rho^{(\varepsilon, \tau)}\right)^{m}-\rho^{(\varepsilon, \tau)} \nabla c^{(\varepsilon, \tau)}\right) \cdot \nabla \phi d x d t \\
& +\varepsilon \int_{\tau}^{T} \int_{\Omega}\left(\Delta r^{(\varepsilon, \tau)} \Delta \phi+\left|\nabla r^{(\varepsilon, \tau)}\right|^{2} \nabla r^{(\varepsilon, \tau)} \cdot \nabla \phi+r^{(\varepsilon, \tau)} \phi\right) d x d t \\
0= & \alpha \int_{\tau}^{T}\left\langle D_{\tau} c^{(\varepsilon, \tau)}, \psi\right\rangle d t+\int_{\tau}^{T} \int_{\Omega}\left(\nabla c^{(\varepsilon, \tau)}+\delta \nabla\left(\rho^{(\varepsilon, \tau)}\right)^{n}\right) \cdot \nabla \psi d x d t \\
& +\int_{\tau}^{T} \int_{\Omega}\left(\rho^{(\varepsilon, \tau)}-c^{(\varepsilon, \tau)}\right) \psi d x d t
\end{aligned}
$$

for all smooth test functions $\phi$ and $\psi$, where $\langle\cdot, \cdot\rangle$ is a dual product. We set $\Omega_{T}=\Omega \times(0, T)$ for given $T>0$. Before we can perform the limit $(\varepsilon, \tau) \rightarrow 0$, we need to prove some uniform bounds in $\varepsilon$ and $\tau$. The following result is a consequence of the discrete entropy estimate of Lemma 10 and the $L^{1}$ bound (14), after integrating with respect to time.

Lemma 11. Let $T>0$ and $1-2 / d<p \leq m$. Then the following uniform bounds hold:

$$
\begin{aligned}
\left\|\rho^{(\varepsilon, \tau)}\right\|_{L^{\infty}\left(0, T ; L^{1}(\Omega) \cap L^{n}(\Omega)\right)}+\left\|\nabla\left(\rho^{(\varepsilon, \tau)}\right)^{p}\right\|_{L^{2}\left(\Omega_{T}\right)} & \leq C, \\
\sqrt{\varepsilon}\left\|r^{(\varepsilon, \tau)}\right\|_{L^{2}\left(0, T ; H^{2}(\Omega)\right)}+\sqrt[4]{\varepsilon}\left\|\nabla r^{(\varepsilon, \tau)}\right\|_{L^{4}\left(\Omega_{T}\right)} & \leq C, \\
\alpha\left\|c^{(\varepsilon, \tau)}\right\|_{L^{\infty}\left(0, T ; L^{2}(\Omega)\right)}+\left\|c^{(\varepsilon, \tau)}\right\|_{L^{2}\left(0, T ; H^{1}(\Omega)\right)} & \leq C,
\end{aligned}
$$

where $C>0$ is here and in the following a generic constant independent of $\varepsilon$ and $\tau$, and $p$ is defined in (10).

Under additional assumptions on the exponents $n$ and $p$, we are able to derive more a priori estimates. 
Lemma 12. Let $p \leq \min \{m, n\}$ and $Q:=n / d+p>1$ and set

$$
s_{1}=\frac{2 Q}{Q+m-p} \in(1,2], \quad s_{2}=\frac{2 Q}{Q+n-p} \in(1,2], \quad s_{3}=\frac{2 Q}{Q+1} \in(1,2) .
$$

Then the following uniform bounds hold:

$$
\begin{aligned}
& \left\|\left(\rho^{(\varepsilon, \tau)}\right)^{p}\right\|_{L^{2}\left(0, T ; H^{1}(\Omega)\right)}+\left\|\rho^{(\varepsilon, \tau)}\right\|_{L^{2 Q}\left(\Omega_{T}\right)} \leq C, \\
& \left\|\left(\rho^{(\varepsilon, \tau)}\right)^{m}\right\|_{L^{s_{1}}\left(0, T ; W^{1, s_{1}}(\Omega)\right)}+\left\|\left(\rho^{(\varepsilon, \tau)}\right)^{n}\right\|_{L^{s_{2}}\left(0, T ; W^{1, s_{2}}(\Omega)\right)} \leq C, \\
& \left\|\rho^{(\varepsilon, \tau)} \nabla c^{(\varepsilon, \tau)}\right\|_{L^{s_{3}}\left(\Omega_{T}\right)} \leq C, \\
& \left\|D_{\tau} \rho^{(\varepsilon, \tau)}\right\|_{L^{\tilde{s}}\left(\tau, T ;\left(H^{3}(\Omega)\right)^{\prime}\right)}+\alpha\left\|D_{\tau} c^{(\varepsilon, \tau)}\right\|_{L^{s}\left(\tau, T ;\left(H^{3}(\Omega)\right)^{\prime}\right)} \leq C,
\end{aligned}
$$

where $s=\min \left\{s_{1}, s_{2}, s_{3}\right\}, \tilde{s}=\min \{s, 4 / 3\}$, and $p$ is defined in (10).

We remark that the condition $Q>1$ is equivalent to $p>1-n / d$, which in particular implies the condition $p>1-2 / d$ as explained in the introduction after (7).

Proof. We set $\rho=\rho^{(\varepsilon, \tau)}$ and $c=c^{(\varepsilon, \tau)}$ to simplify the notation. By the Poincaré inequality, we find that

$$
\left\|\rho^{p}\right\|_{L^{2}\left(\Omega_{T}\right)}^{2}=\int_{0}^{T}\left\|\rho^{p}\right\|_{L^{2}(\Omega)}^{2} d t \leq C \int_{0}^{T}\left\|\nabla \rho^{p}\right\|_{L^{2}(\Omega)}^{2} d t+C \int_{0}^{T}\left\|\rho^{p}\right\|_{L^{1}(\Omega)}^{2} d t .
$$

Since $\rho$ is uniformly bounded in $L^{\infty}\left(0, T ; L^{n}(\Omega)\right)$ and since we have assumed that $p \leq n$, the right-hand side of the above inequality is uniformly bounded. This shows the uniform bound for $\rho^{p}$ in $L^{2}\left(0, T ; H^{1}(\Omega)\right)$. Next, the Gagliardo-Nirenberg inequality with $\theta=p / Q<$ 1 gives

$$
\begin{aligned}
\|\rho\|_{L^{2 Q\left(\Omega_{T}\right)}}^{2 Q} & =\left\|\rho^{p}\right\|_{L^{2 Q / p}\left(\Omega_{T}\right)}^{2 Q / p} \leq C \int_{0}^{T}\left\|\rho^{p}\right\|_{H^{1}(\Omega)}^{2 Q \theta / p}\left\|\rho^{p}\right\|_{L^{n / p}(\Omega)}^{2 Q(1-\theta) / p} d t \\
& \leq C\|\rho\|_{L^{\infty}\left(0, T ; L^{n}(\Omega)\right)}^{2 Q(1-\theta)} \int_{0}^{T}\left\|\rho^{p}\right\|_{H^{1}(\Omega)}^{2} d t \leq C .
\end{aligned}
$$

This shows (21).

For the proof of (22), we observe that $s_{1}>1$ is equivalent to $n / d+n>1$, which is true since $n>1$, and that $s_{1} \leq 2$ is equivalent to $p \leq m$, which holds by assumption. Hence $s_{1} \in(1,2]$. Let first $s_{1}<2$. We apply the Hölder inequality with exponents $\gamma=2 / s_{1}>1$ and $\gamma^{\prime}=2 /\left(2-s_{1}\right)$ :

$$
\begin{aligned}
\left\|\nabla\left(\rho^{m}\right)\right\|_{L^{s_{1}\left(\Omega_{T}\right)}}^{s_{1}} & =\left(\frac{m}{p}\right)^{s_{1}} \int_{0}^{T} \int_{\Omega} \rho^{(m-p) s_{1}}\left|\nabla \rho^{p}\right|^{s_{1}} d x d t \\
& \leq C\|\rho\|_{L^{2 Q}\left(\Omega_{T}\right)}^{(m-p) s_{1}}\left\|\nabla \rho^{p}\right\|_{L^{2}\left(\Omega_{T}\right)}^{s_{1}} \leq C,
\end{aligned}
$$

because of (21). If $s_{1}=2$, it follows that $m=p$, and the conclusion still holds. The estimate for $\rho^{m}$ is shown in a similar way by applying the Hölder inequality with exponents $\gamma=2 / s_{1}>1$ (if $\left.s_{2}<2\right)$ and $\gamma^{\prime}=2 /\left(2-s_{1}\right)$ to $\rho^{m}=\rho^{m-p} \rho^{p}$. Hence, $\rho^{m}$ is uniformly bounded in $L^{s_{1}}\left(0, T ; W^{1, s_{1}}(\Omega)\right)$. 
The estimates for $\nabla\left(\rho^{n}\right)$ and $\rho^{n}$ in $L^{s_{2}}\left(\Omega_{T}\right)$ are proved analogously by replacing $s_{1}$ by $s_{2}$. The relation $s_{2} \leq 2$ is equivalent to $p \leq n$, and $s_{2}>1$ is equivalent to $n / d+m>1$, which is true since $n / d+m \geq n / d+p>1$ by assumption. This proves (22).

Estimate (23) is again a consequence of the Hölder inequality, with exponents $\alpha=2 / s_{3}$ and $\alpha^{\prime}=2 /\left(2-s_{3}\right)$ :

$$
\|\rho \nabla c\|_{L^{s_{3}\left(\Omega_{T}\right)}} \leq\|\rho\|_{L^{2 s_{3} /\left(2-s_{3}\right)\left(\Omega_{T}\right)}}\|\nabla c\|_{L^{2}\left(\Omega_{T}\right)}=\|\rho\|_{L^{2 Q}\left(\Omega_{T}\right)}\|\nabla c\|_{L^{2}\left(\Omega_{T}\right)} \leq C .
$$

We turn now to the estimates for the discretized time derivatives. Let $\phi \in L^{\tilde{s}^{\prime}}(0, T$; $\left.H^{3}(\Omega)\right)$, where $s^{\prime}=s /(s-1) \geq 2$ and $\tilde{s}^{\prime}=\max \left\{s^{\prime}, 4\right\}$. Then, using $1<s \leq 2$ and (19), $(22)$, and (23),

$$
\begin{aligned}
& \left|\int_{\tau}^{T}\left\langle D_{\tau} \rho, \phi\right\rangle d t\right| \\
& =\left|-\int_{\tau}^{T} \int_{\Omega}\left(\nabla\left(\rho^{m}\right)-\rho \nabla c\right) \cdot \nabla \phi d x d t-\varepsilon \int_{\tau}^{T} \int_{\Omega}\left(\Delta r \Delta \phi+|\nabla r|^{2} \nabla r \cdot \nabla \phi+r \phi\right) d x d t\right| \\
& \leq\left(\left\|\nabla\left(\rho^{m}\right)\right\|_{L^{s}\left(\Omega_{T}\right)}+\|\rho \nabla c\|_{L^{s}\left(\Omega_{T}\right)}\right)\|\nabla \phi\|_{L^{s^{\prime}}\left(\Omega_{T}\right)} \\
& \quad+\varepsilon\|\Delta r\|_{L^{2}\left(\Omega_{T}\right)}\|\Delta \phi\|_{L^{2}\left(\Omega_{T}\right)}+\varepsilon\|\nabla r\|_{L^{4}\left(\Omega_{T}\right)}^{3}\|\nabla \phi\|_{L^{4}\left(\Omega_{T}\right)}+\varepsilon\|r\|_{L^{2}\left(\Omega_{T}\right)}\|\phi\|_{L^{2}\left(\Omega_{T}\right)} \\
& \quad \leq C\|\phi\|_{\left.L^{\tilde{s}^{\prime}\left(0, T ; H^{3}(\Omega)\right.}\right)} .
\end{aligned}
$$

Furthermore, since $\rho \in L^{2 Q}\left(\Omega_{T}\right) \subset L^{2}\left(\Omega_{T}\right)$, we obtain

$$
\begin{aligned}
\left|\int_{\tau}^{T}\left\langle D_{\tau} c, \phi\right\rangle d t\right|= & \left|-\int_{\tau}^{T} \int_{\Omega}\left(\nabla c+\delta \nabla\left(\rho^{n}\right)\right) \cdot \nabla \phi d x d t+\int_{\tau}^{T} \int_{\Omega}(\rho-c) \phi d x d t\right| \\
\leq & \left(\|\nabla c\|_{L^{s}\left(\Omega_{T}\right)}+\delta\left\|\nabla\left(\rho^{n}\right)\right\|_{L^{s}\left(\Omega_{T}\right)}\right)\|\nabla \phi\|_{L^{s^{\prime}}\left(\Omega_{T}\right)} \\
& +\left(\|\rho\|_{L^{2}\left(\Omega_{T}\right)}+\|c\|_{L^{2}\left(\Omega_{T}\right)}\right)\|\phi\|_{L^{2}\left(\Omega_{T}\right)} \\
\leq & C\|\phi\|_{L^{s^{\prime}}\left(0, T ; H^{3}(\Omega)\right)} .
\end{aligned}
$$

using (20), (21), and (22). Thus we have proved (24).

3.3. The limit of vanishing approximation parameters. We show first the strong convergence of the sequence $\left(\rho^{(\varepsilon, \tau)}\right)$.

Lemma 13. Let the assumptions of Lemma 12 hold. Then, up to a subsequence,

$$
\rho^{(\varepsilon, \tau)} \rightarrow \rho \text { strongly in } L^{2 r}\left(0, T ; L^{2 Q}(\Omega)\right),
$$

for all $r<Q$, where $Q=n / d+p>1$.

Proof. First, consider $p<1$. We show that $\left(\rho^{(\varepsilon, \tau)}\right)$ is bounded in $L^{\ell}\left(0, T ; W^{1, \ell}(\Omega)\right)$, where $\ell=2 Q /(Q+1-p)>1$. Notice that $1<\ell<2$ since the former inequality is equivalent to $Q>1-p$ which is true since $Q>1$ by assumption, and the latter property is equivalent to $p<1$. We apply the Hölder inequality to $\nabla \rho^{(\varepsilon, \tau)}=(1 / p)\left(\rho^{(\varepsilon, \tau)}\right)^{1-p} \nabla\left(\rho^{(\varepsilon, \tau)}\right)^{p}$ yielding

$$
\begin{aligned}
\left\|\nabla \rho^{(\varepsilon, \tau)}\right\|_{L^{\ell}\left(\Omega_{T}\right)} & \leq C\left\|\left(\rho^{(\varepsilon, \tau)}\right)^{1-p}\right\|_{L^{2 \ell /(2-\ell)}\left(\Omega_{T}\right)}\left\|\nabla\left(\rho^{(\varepsilon, \tau)}\right)^{p}\right\|_{L^{2}\left(\Omega_{T}\right)} \\
& =C\left\|\rho^{(\varepsilon, \tau)}\right\|_{L^{2 Q}\left(\Omega_{T}\right)}^{1-p}\left\|\nabla\left(\rho^{(\varepsilon, \tau)}\right)^{p}\right\|_{L^{2}\left(\Omega_{T}\right)},
\end{aligned}
$$


PREVENTING BLOW UP IN THE KELLER-SEGEL MODEL

since $2 \ell(1-p) /(2-\ell)=2 Q$. Thus, taking into account the bound for $\left(\rho^{(\varepsilon, \tau)}\right)$ in $L^{2 Q}\left(\Omega_{T}\right)$, by $(21)$, this proves the desired bound.

Next, we claim that the embedding $W^{1, \ell}(\Omega) \hookrightarrow L^{2 Q}(\Omega)$ is compact. This is the case if $1-d / \ell>-d /(2 Q)$, which is equivalent to $2 n / d+m>1$. In order to show this inequality, we observe that the assumption $p>1-2 / d$ is equivalent to $n+m>3-4 / d$. If $d=2$, this implies that $2 n / d+m=n+m>1$; if $d=3$, we find that

$$
\frac{2 n}{d}+m=\frac{2}{3}(n+m)+\frac{m}{3}>\frac{2}{3}(n+m)>\frac{2}{3} \cdot \frac{5}{3}>1 \text {. }
$$

Hence, $1-d / \ell>-d /(2 Q)$ is satisfied for $d \leq 3$. In view of the bound for the discrete time derivative of $\left(\rho^{(\varepsilon, \tau)}\right)$, see (24), Lemma 7 (take $p=1$ in the lemma) implies the existence of a subsequence of $\left(\rho^{(\varepsilon, \tau)}\right)$ (not relabeled) such that $(25)$ holds.

Finally, if $p \geq 1$, we can apply Lemma 7 to conclude the strong convergence result.

The estimates of Lemmas 11 and 12 imply the existence of a subsequence of $\left(\rho^{(\varepsilon, \tau)}\right)$, which is not relabeled, such that, as $(\varepsilon, \tau) \rightarrow 0$,

$$
\begin{aligned}
c^{(\varepsilon, \tau)} \rightarrow c & \text { weakly in } L^{2}\left(0, T ; H^{1}(\Omega)\right), \\
\left(\rho^{(\varepsilon, \tau)}\right)^{m} \rightarrow z_{1} & \text { weakly in } L^{s_{1}}\left(0, T ; W^{1, s_{1}}(\Omega)\right), \\
\left(\rho^{(\varepsilon, \tau)}\right)^{n} \rightarrow z_{2} & \text { weakly in } L^{s_{2}}\left(0, T ; W^{1, s_{2}}(\Omega)\right), \\
\rho^{(\varepsilon, \tau)} \nabla c^{(\varepsilon, \tau)} \rightarrow z_{3} & \text { weakly in } L^{s_{3}}\left(0, T ; W^{1, s_{3}}(\Omega)\right), \\
D_{\tau} \rho^{(\varepsilon, \tau)} \rightarrow \partial_{t} \rho & \text { weakly in } L^{\tilde{s}}\left(0, T ;\left(H^{3}(\Omega)\right)^{\prime}\right), \\
D_{\tau} c^{(\varepsilon, \tau)} \rightarrow \partial_{t} c & \text { weakly in } L^{s}\left(0, T ;\left(H^{3}(\Omega)\right)^{\prime}\right) \text { if } \alpha>0, \\
\varepsilon r^{(\varepsilon, \tau)} \rightarrow 0 & \text { weakly in } L^{2}\left(0, T ; H^{2}(\Omega)\right), \\
\varepsilon\left|\nabla r^{(\varepsilon, \tau)}\right|^{2} \nabla r^{(\varepsilon, \tau)} \rightarrow 0 & \text { weakly in } L^{4 / 3}\left(\Omega_{T}\right) .
\end{aligned}
$$

The limits of the nonlinearities are easily identified since (a subsequence of $)\left(\rho^{(\varepsilon, \tau)}\right)$ converges strongly in $L^{2 Q}\left(\Omega_{T}\right)$, where $Q>1$. Hence, up to a subsequence, $\rho^{(\varepsilon, \tau)} \rightarrow \rho$ a.e. in $\Omega_{T}$ and $\left(\rho^{(\varepsilon, \tau)}\right)^{m} \rightarrow \rho^{m},\left(\rho^{(\varepsilon, \tau)}\right) \rightarrow \rho^{n}$ a.e., implying that $z_{1}=\rho^{m}, z_{2}=\rho^{n}$. Moreover, the strong convergence of $\left(\rho^{(\varepsilon, \tau)}\right)$ in $L^{2}\left(\Omega_{T}\right)$ and the weak convergence of $\nabla c^{(\varepsilon, \tau)}$ in $L^{2}\left(\Omega_{T}\right)$ give the weak convergence of $\left(\rho^{(\varepsilon, \tau)} \nabla c^{(\varepsilon, \tau)}\right)$ to $\rho \nabla c$ in $L^{1}\left(\Omega_{T}\right)$, implying that $z_{3}=\rho \nabla c$.

The above convergence results are sufficient to pass to the limit $(\varepsilon, \tau) \rightarrow 0$ in (16)-(17) leading to (1)-(2). The Neumann boundary conditions are satisfied in the weak sense, and the initial conditions hold in the sense of $L^{\tilde{s}}\left(0, T ;\left(H^{3}(\Omega)\right)^{\prime}\right)$. Since in the limiting equation the regularizing terms vanish, test functions in $L^{s^{\prime}}\left(0, T ; W^{1, s^{\prime}}(\Omega)\right)$ are sufficient to obtain the boundedness of the diffusion and drift terms. A density argument now completes the proof.

\section{The PARABOLIC-ELLIPTIC SYSTEM}

The parabolic-elliptic system corresponding to (2) is given by

$$
\partial_{t} \rho=\Delta \rho^{m}-\operatorname{div}(\rho \nabla c), \quad 0=\Delta c+\delta \Delta \rho^{n}+\rho-c,
$$


subject to the no-flux boundary conditions

$$
\left(\nabla \rho^{m}-\rho \nabla c\right) \cdot \nu=0, \quad \nabla\left(c+\delta \rho^{n}\right) \cdot \nu=0 \quad \text { on } \partial \Omega, t>0,
$$

and the initial condition

$$
\rho(\cdot, 0)=\rho_{0} \quad \text { in } \Omega .
$$

Similarly as in [14] we introduce a new unknown corresponding to the diffusion terms in the second equation, $v=c+\delta \rho^{n}$, and rewrite system (26) in terms of $\rho$ and $v$ :

$$
\begin{aligned}
\partial_{t} \rho & =\Delta\left(\rho^{m}+\delta \frac{n}{n+1} \rho^{n+1}\right)-\operatorname{div}(\rho \nabla v), \\
0 & =\Delta v+\rho+\delta \rho^{n}-v,
\end{aligned}
$$

subject to the no-flux boundary conditions and initial data (28). In the case $n=1$ one simply obtains the Keller-Segel model with nonlinear diffusion, which is known to prevent blow up [4, 21, 22]. Here, the situation is different, since for $n>1$ we obtain additionally a nonlinear growth term in the equation for $v$. We will show that nevertheless this system satisfies the properties used by Kowalczyk in [21] to obtain an $L^{\infty}\left(\Omega_{T}\right)$ bound for $\rho$. The difference here is that instead of the conservation of mass we have to make use of the uniform boundedness of $\|\rho\|_{L^{n}(\Omega)}$, which only holds for finite times.

The main advantage of the parabolic-elliptic system is that it allows for another entropy, since we will show that we can use powers of $\rho$ as test functions in the elliptic equation, see Remark 2.

Lemma 14. Let $(\rho, v)$ be the solution of the parabolic-elliptic system (26) constructed in Theorem 1 with $\rho_{0} \in L^{n}(\Omega)$ and $\rho_{0} \geq 0$. Then

$$
\rho \geq 0, \quad v \geq 0 \quad \text { a.e. in } \Omega_{T}
$$

and $\rho$ satisfies the additional entropy estimate

$$
\sup _{t \in(0, T)} \int_{\Omega} \frac{\rho(\cdot, t)^{n}}{n-1} d x+\frac{n m}{p^{2}}\left\|\nabla \rho^{p}\right\|_{L^{2}\left(\Omega_{T}\right)}^{2}+\frac{\delta}{2}\left\|\nabla \rho^{n}\right\|_{L^{2}\left(\Omega_{T}\right)}^{2} \leq C .
$$

Proof. We start again from the regularized problem to derive the nonnegativity of $v$ and the additional entropy estimate rigorously. As in Section 3 we skip the index $k$ :

$$
\begin{aligned}
\int_{\Omega} D_{\tau} \rho \phi d x= & -\int_{\Omega} \nabla\left(\rho^{m}+\delta \frac{n}{n+1} \rho^{n+1}\right) \cdot \nabla \phi d x+\int_{\Omega} \rho \nabla v \cdot \nabla \phi d x \\
& -\varepsilon \int_{\Omega}\left(\Delta r \Delta \phi+|\nabla r|^{2} \nabla r \cdot \nabla \phi+r \phi\right) d x \\
0= & -\int_{\Omega} \nabla v \cdot \nabla \psi d x+\int_{\Omega}\left(\rho+\delta \rho^{n}\right) \psi d x-\int_{\Omega} v \psi d x
\end{aligned}
$$

for appropriate test functions $\phi$ and $\psi$. The existence of a global weak solution is proven in Proposition 9. The a priori nonnegativity of $v$ follows from a standard argument by testing the equation for $v$ with $v^{-}=\min \{0, v\}$, see, e.g., [21]. The nonnegativity is clearly preserved when performing the limit of vanishing parameters. 
To derive the additional energy estimate we first use $r=\frac{n}{n-1} \rho^{n-1}$ as a test function in the equation for $\rho$, integrate in time, and insert the elliptic equation for $v$ :

$$
\begin{aligned}
& \int_{\Omega} \frac{\rho^{n}}{n-1} d x-\int_{\Omega} \frac{\rho_{0}^{n}}{n-1} d x+\frac{n m}{p^{2}}\left\|\nabla \rho^{p}\right\|_{L^{2}\left(\Omega_{T}\right)}^{2}+\delta\left\|\nabla \rho^{n}\right\|_{L^{2}\left(\Omega_{T}\right)}^{2} \\
& \quad \leq \int_{\Omega_{T}} \nabla v \cdot \nabla \rho^{n} d x d t-\varepsilon\left(\|\Delta r\|_{L^{2}\left(\Omega_{T}\right)}^{2}+\|\nabla r\|_{L^{4}\left(\Omega_{T}\right)}^{4}+\|r\|_{L^{2}\left(\Omega_{T}\right)}^{2}\right) \\
& \quad \leq \int_{\Omega_{T}} \nabla v \cdot \nabla \rho^{n} d x d t=\int_{\Omega_{T}} \rho^{n+1} d x d t+\delta \int_{\Omega_{T}} \rho^{2 n} d x d t-\int_{\Omega_{T}} v \rho^{n} d x d t \\
& \quad \leq C+\frac{\delta}{2}\left\|\nabla \rho^{n}\right\|_{L^{2}\left(\Omega_{T}\right)}^{2} .
\end{aligned}
$$

Here, we have used the nonnegativity of $\rho$ and $v$ and the uniform boundedness of $\|\rho\|_{L^{n}(\Omega)}$ (with respect to $k$ ) together with the Gagliardo-Nirenberg inequalities in the following way:

$$
\begin{aligned}
\int_{\Omega} \rho^{n+1} d x & =\left\|\rho^{n}\right\|_{L^{(n+1) / n}(\Omega)}^{(n+1) / n} \leq C\left\|\nabla \rho^{n}\right\|_{L^{2}(\Omega)}^{(n+1) \theta_{1} / n}\left\|\rho^{n}\right\|_{L^{1}(\Omega)}^{(n+1)\left(1-\theta_{1}\right) / n}+\left\|\rho^{n}\right\|_{L^{1}(\Omega)}^{(n+1) / n} \\
& \leq C\left\|\nabla \rho^{n}\right\|_{L^{2}(\Omega)}^{(n+1) \theta_{1} / n}+C \leq \frac{\delta}{4}\left\|\nabla \rho^{n}\right\|_{L^{2}(\Omega)}^{2}+C(\delta),
\end{aligned}
$$

where $\theta_{1}=2 d /((n+1)(2+d)) \in(0,1)$, and for $\theta_{2}=d /(d+2)$, we obtain the bound

$$
\int_{\Omega} \rho^{2 n} d x=\left\|\rho^{n}\right\|_{L^{2}(\Omega)}^{2} \leq C\left\|\nabla \rho^{n}\right\|_{L^{2}(\Omega)}^{2 \theta_{2}}\left\|\rho^{n}\right\|_{L^{1}(\Omega)}^{2\left(1-\theta_{2}\right)}+\left\|\rho^{n}\right\|_{L^{1}(\Omega)}^{2} \leq \frac{\delta}{4}\left\|\nabla \rho^{n}\right\|_{L^{2}(\Omega)}^{2}+C(\delta) .
$$

Since the constant $C(\delta)$ is independent of $(\varepsilon, \tau)$, we can perform the limit of vanishing parameters, which completes the proof.

Theorem 15. Let $\rho_{0} \in L^{\infty}(\Omega)$, where $\Omega \subset \mathbb{R}^{d}$ is a bounded domain $(1 \leq d \leq 3)$ with $\partial \Omega \in C^{1,1}$, and let the assumptions (5) hold. Then the parabolic-elliptic system (26)-(28) has a global weak solution satisyfing

$$
\|\rho\|_{L^{\infty}\left(0, T ; L^{\infty}(\Omega)\right)}+\|c\|_{L^{\infty}\left(0, T ; L^{\infty}(\Omega)\right)} \leq C(T)
$$

for any $T>0$.

In particular, test functions in $L^{2}\left(0, T ; H^{1}(\Omega)\right)$ are admissible for (26).

Proof. The iterative method of Alikakos used by Kowalczyk to derive an $L^{\infty}\left(\Omega_{T}\right)$ bound for $\rho$ requires test functions of the form $\rho^{q}$ for $q \geq n$ as well as the uniform boundedness of $\nabla v$. Thus, we prove first that $\nabla v \in L^{\infty}\left(\Omega_{T}\right)$ and $\rho \in L^{\infty}\left(0, T ; L^{q}(\Omega)\right)$ for suitable $q>1$.

Step 1: Proof of $v \in L^{\infty}\left(0, T ; W^{1, \infty}(\Omega)\right)$. If $\rho \in L^{\infty}\left(0, T ; L^{3 n+1}(\Omega)\right)$, then elliptic regularity for

$$
-\Delta v+v=\rho+\delta \rho^{n} \quad \text { in } \Omega, \quad \nabla v \cdot \nu=0 \quad \text { in } \partial \Omega
$$

implies that $v \in L^{\infty}\left(0, T ; W^{2,3+1 / n}(\Omega)\right)$ (see, e.g., [11], p. 126), and hence, by Sobolev embedding, $v \in L^{\infty}\left(0, T ; W^{1, \infty}(\Omega)\right)$. In order to show that $\rho \in L^{\infty}\left(0, T ; L^{3 n+1}(\Omega)\right)$, we 
employ in the regularized equation $(31)$ the test function $(3 n+1) \rho^{3 n}$ and integrate in time:

$$
\begin{aligned}
& \int_{\Omega} \rho^{3 n+1} d x-\int_{\Omega} \rho_{0}^{3 n+1} d x+2 a\left\|\nabla \rho^{(m+3 n) / 2}\right\|_{L^{2}\left(\Omega_{T}\right)}^{2}+2 \delta b\left\|\nabla \rho^{2 n+1 / 2}\right\|_{L^{2}\left(\Omega_{T}\right)}^{2} \\
& \quad+(3 n+1) \varepsilon\left(\int_{\Omega_{T}} \Delta r \Delta \rho^{3 n}+|\nabla r|^{2} \nabla r \cdot \nabla \rho^{3 n}+r \rho^{3 n}\right) d x d t \\
& \leq 3 n \int_{\Omega_{T}} \nabla \rho^{3 n+1} \cdot \nabla v d x d t \\
& \quad=3 n \int_{\Omega_{T}} \rho^{3 n+2} d x d t+3 \delta n \int_{\Omega_{T}} \rho^{4 n+1} d x d t-3 n \int_{\Omega_{T}} \rho^{3 n+1} v d x d t \\
& \leq 4 \delta n \int_{\Omega_{T}} \rho^{4 n+1} d x d t+C,
\end{aligned}
$$

where we have used the nonnegativity of $\rho$ and $v$, Hölder's inequality, and $3 n+2<4 n+1$. Moreover, we have set $a=6 m n(3 n+1) /(m+3 n)^{2}$ and $b=6 n^{2}(3 n+1) /(4 n+1)^{2}$. We apply the Gagliardo-Nirenberg inequality with $\theta=d /(d+2)$ and Hölder's and Young's inequalities to estimate

$$
\begin{aligned}
\int_{\Omega} \rho^{4 n+1} d x & =\left\|\rho^{2 n+1 / 2}\right\|_{L^{2}(\Omega)}^{2} \leq C\left\|\nabla \rho^{2 n+1 / 2}\right\|_{L^{2}(\Omega)}^{2 \theta}\left\|\rho^{2 n+1 / 2}\right\|_{L^{1}(\Omega)}^{2(1-\theta)}+\left\|\rho^{2 n+1 / 2}\right\|_{L^{1}(\Omega)}^{2} \\
& \leq \frac{b}{4 n}\left\|\nabla \rho^{2 n+1 / 2}\right\|_{L^{2}(\Omega)}^{2}+C\left\|\rho^{2 n+1 / 2}\right\|_{L^{1}(\Omega)}^{2} .
\end{aligned}
$$

The last summand is estimated by interpolating, for any $\beta>0$,

$$
\left\|\rho^{2 n+1 / 2}\right\|_{L^{1}(\Omega)}^{2} \leq\left\|\rho^{(3 n+1) / 2}\right\|_{L^{2}(\Omega)}^{2}\left\|\rho^{n}\right\|_{L^{1}(\Omega)} \leq C \int_{\Omega} \rho^{3 n+1} d x \leq \beta \int_{\Omega} \rho^{4 n+1} d x+C(\beta),
$$

since $\rho \in L^{\infty}\left(0, T ; L^{n}(\Omega)\right)$. Finally, combining the above estimates and by choosing $\beta$ appropriately, we obtain

$$
\int_{\Omega_{T}} \rho^{4 n+1} d x d t \leq \frac{b}{4 n}\left\|\nabla \rho^{2 n+1 / 2}\right\|_{L^{2}\left(\Omega_{T}\right)}^{2}+C .
$$

It remains to bound from below the terms arising from the regularization:

$$
\begin{aligned}
\varepsilon \int_{\Omega_{T}}\left(\Delta r \Delta \rho^{3 n}+|\nabla r|^{2} \nabla r \cdot \nabla \rho^{3 n}+r \rho^{3 n}\right) d x d t \\
\geq 3 \varepsilon \int_{\Omega_{T}}\left(\rho^{2 n+1}(\Delta r)^{2}+2\left(1+\frac{1}{2 n}\right) \rho^{n+2}|\nabla r|^{2} \Delta r+\rho^{2 n+1}|\nabla r|^{4}\right) d x d t \\
=3 \varepsilon \int_{\Omega_{T}}\left(\rho^{n+1 / 2} \Delta r+\left(1+\frac{1}{2 n}\right) \rho^{3 / 2}|\nabla r|^{2}\right)^{2} d x d t \\
\quad+3 \varepsilon \int_{\Omega_{T}} \rho^{3}\left(\rho^{2(n-1)}-\left(1+\frac{1}{2 n}\right)^{2}\right)|\nabla r|^{4} d x d t
\end{aligned}
$$


PREVENTING BLOW UP IN THE KELLER-SEGEL MODEL

$$
\begin{aligned}
& \geq \varepsilon \int_{\left\{\rho^{n-1} \leq 1+1 / 2 n\right\}} \rho^{3}\left(\rho^{2(n-1)}-\left(1+\frac{1}{2 n}\right)^{2}\right)|\nabla r|^{4} d x d t \\
& \geq-\varepsilon C\|\nabla r\|_{L^{4}\left(\Omega_{T}\right)}^{4} \geq-C,
\end{aligned}
$$

where $C$ is independent of $(\varepsilon, \tau)$ due to the basic entropy estimate for the regularized system. Thus, we obtain in the limit $(\varepsilon, \tau) \rightarrow 0$ for the weak solution of (26),

$$
\int_{\Omega} \rho^{3 n+1} d x+\delta b\left\|\nabla \rho^{2 n+1 / 2}\right\|_{L^{2}\left(\Omega_{T}\right)}^{2} \leq C .
$$

This implies that $\rho \in L^{\infty}\left(0, T ; L^{3 n+1}(\Omega)\right)$, which proves the claim.

Step 2: Test functions in $L^{2}\left(0, T ; H^{1}(\Omega)\right)$ are admissible. We have to verify that $\nabla \rho^{m} \in$ $L^{2}\left(0, T ; L^{2}(\Omega)\right)$. We recall that the restrictions on the exponents are $n-1 \leq m \leq n+1$ or, equivalently, $p \leq m \leq n+1 \leq 2 n+1 / 2$. Therefore, in view of Step 1 , we can interpolate

$$
\begin{aligned}
\frac{p^{2}}{m^{2}} \int_{\Omega_{T}}\left|\nabla \rho^{m}\right|^{2} d x d t & =\int_{\Omega_{T}} \rho^{2(m-p)}\left|\nabla \rho^{p}\right|^{2} d x d t \\
& \leq \int_{\{\rho \leq 1\}}\left|\nabla \rho^{p}\right|^{2} d x d t+\int_{\{\rho \geq 1\}} \rho^{2(2 n+1 / 2-p)}\left|\nabla \rho^{p}\right|^{2} d x d t \\
& \leq\left\|\nabla \rho^{p}\right\|_{L^{2}\left(\Omega_{T}\right)}^{2}+C\left\|\nabla \rho^{2 n+1 / 2}\right\|_{L^{2}\left(\Omega_{T}\right)}^{2} \leq C .
\end{aligned}
$$

Step 3: We now proceed to make the estimates derived by Kowalczyk rigorous. To this end, we will use powers of the cut-off functions $\rho_{K}=\min \{\rho, K\}$ as test functions. Due to (30), $\rho_{K}^{q} \in L^{2}\left(0, T ; H^{1}(\Omega)\right)$ for any $q \geq n$ and according to Step 2, it is an admissible test function. Let us introduce the notation

$$
w(x, t)=\left(\rho_{K}-k\right)_{+} \quad \text { for some } k>0 .
$$

We test the equation for the cell density with $(q+1) w^{q}$, where $q \geq n$ :

$$
\begin{aligned}
\partial_{t} \int_{\Omega} w^{q+1} d x= & -\delta(q+1) \int_{\Omega} \nabla \rho^{n} \cdot \nabla w^{q} d x-(q+1) \int_{\Omega} \nabla \rho^{m} \cdot \nabla w^{q} d x \\
& +(q+1) \int_{\Omega} \rho \nabla w^{q} \cdot \nabla v d x \\
\leq & -\delta n(q+1) k^{n-1} \int_{\Omega} \nabla \rho \cdot \nabla w^{q} d x-m q(q+1) \int_{\Omega} \rho^{m-1} w^{q-1}|\nabla \rho|^{2} d x \\
& +2 q\|\nabla v\|_{L^{\infty}(\Omega)}\left(\int_{\Omega} w^{(q+1) / 2}\left|\nabla w^{(q+1) / 2}\right| d x+k \int_{\Omega} w^{(q-1) / 2}\left|\nabla w^{(q+1) / 2}\right| d x\right)
\end{aligned}
$$

Neglecting the second term on the right-hand side and employing Young's inequality to the last two terms, we arrive at

$$
\partial_{t} \int_{\Omega} w^{q+1} d x \leq-\delta \frac{2 n q}{q+1} k^{n-1} \int_{\Omega}\left|\nabla w^{(q+1) / 2}\right|^{2} d x+C q(q+1) \int_{\Omega} w^{q+1} d x+C q(q+1),
$$


where $C>0$ depends on, e.g., $\delta$ and $\|\nabla v\|_{L^{\infty}(\Omega)}$. Starting from this inequality, Kowalczyk [21] employed the iterative method of Alikakos to obtain

$$
\|w(\cdot, t)\|_{L^{\infty}(\Omega)} \leq C\left(\left\|w_{0}\right\|_{L^{\infty}(\Omega)}\right) \leq C\left(\left\|\rho_{0}\right\|_{L^{\infty}(\Omega)}\right) \text { for all } 0<t<T .
$$

Hence, for $\rho_{0} \in L^{\infty}\left(\Omega_{T}\right)$, we have $w=\left(\rho_{K}-k\right)_{+} \in L^{\infty}\left(\Omega_{T}\right)$ for any $K, k>0$ with an independent bound for the norm. We can let $K \rightarrow \infty$ to deduce

$$
\|\rho(\cdot, t)\|_{L^{\infty}(\Omega)} \leq C\left(\left\|\rho_{0}\right\|_{L^{\infty}(\Omega)}\right) \text { for all } 0<t<T,
$$

which finishes the proof.

\section{LONG-TIME BEHAVIOUR}

The (modified) Keller-Segel system possesses the constant homogeneous steady state $\rho^{*}=c^{*}=M /$ meas $(\Omega)$. Let us consider the following system, which is equivalent to (1)-(2):

$$
\begin{aligned}
\partial_{t} \rho & =\Delta \rho^{m}-\operatorname{div}(\rho \nabla c), \\
\alpha \partial_{t} c & =\Delta c+\delta \Delta \rho^{n}+\rho-\rho^{*}-\left(c-c^{*}\right) \quad \text { in } \Omega, t>0 .
\end{aligned}
$$

In the case of linear diffusion terms, $m=n=1$, the decay of the relative entropy yields the convergence of the solution towards the homogeneous steady state for large enough $\delta$ if $d=2$ [14]. The corresponding relative entropy for the system under consideration with nonlinear diffusion is

$$
E^{*}(t)=\int_{\Omega}\left[\frac{\left(\rho-\rho^{*}\right)^{n}}{n-1}+\alpha \frac{\left(c-c^{*}\right)^{2}}{2 \delta}\right] d x .
$$

Notice that the nonnegativity of $E^{*}$ is only guaranteed if $n$ is an even integer. In particular, $E^{*}$ is not well defined for general real $n>1$, since $\rho-\rho^{*}$ may be negative. Formally, testing (32)-(33) with $\left(n\left(\rho-\rho^{*}\right)^{n-1} /(n-1),\left(c-c^{*}\right) / \delta\right)$ we obtain the evolution equation for the relative entropy

$$
\begin{aligned}
\frac{d E^{*}}{d t} & +m n \int_{\Omega} \rho^{m-1}\left(\rho-\rho^{*}\right)^{n-2}|\nabla \rho|^{2} d x+\frac{1}{\delta}\left\|c-c^{*}\right\|_{H^{1}(\Omega)}^{2} \\
& =n \int_{\Omega} \rho\left(\rho-\rho^{*}\right)^{n-2} \nabla c \cdot \nabla \rho d x-n \int_{\Omega} \rho^{n-1} \nabla \rho \cdot \nabla c d x+\frac{1}{\delta} \int_{\Omega}\left(\rho-\rho^{*}\right)\left(c-c^{*}\right) d x .
\end{aligned}
$$

We see that for general values $n>1$, also the entropy dissipation terms are not necessarily nonnegative. Moreover, the chemotactic drift term and the term arising from the crossdiffusion perturbation cancel out only if $n=2$. These comments motivate us to consider the case $n=2$ only. We recall that, due to (5), $n=2$ implies that $1 \leq m \leq 3$. Thus we restrict us to the special case

$$
m=1, \quad n=2,
$$

for which the global existence of a weak solution is guaranteed. This choice allows us to show the decay of the solution to the homogeneous steady state for certain values of $\delta$. For the fully parabolic system we need additionally a smoothness assumption on the solution, since the weak solution, obtained in Theorem 1, cannot be used as a test function (in 
contrast to the parabolic-elliptic system), see Remark 2. Notice that we cannot start from the regularized problem, since there the mass of $\rho$ is not conserved, hence the system does not possesses a constant homogeneous steady state.

Now, we are in the position to prove Proposition 4 (see the introduction).

Proof. Step 1: Decay of the relative entropy. We wish to employ $\left(2\left(\rho-\rho^{*}\right),\left(c-c^{*}\right) / 2 \delta\right)$ as test function in (32)-(33), which is allowed if $\alpha=0$. On the other hand, when $\alpha=1$, we need the smoothness assumption.

Since $n=2$, the evolution equation for the relative entropy

$$
E^{*}(t)=\int_{\Omega}\left[\left(\rho-\rho^{*}\right)^{2}+\alpha \frac{\left(c-c^{*}\right)^{2}}{2 \delta}\right] d x
$$

reduces to

$$
\frac{d E^{*}}{d t}+2\|\nabla \rho\|_{L^{2}(\Omega)}^{2}+\frac{1}{\delta}\left\|c-c^{*}\right\|_{H^{1}(\Omega)}^{2}=\frac{1}{\delta} \int_{\Omega}\left(\rho-\rho^{*}\right)\left(c-c^{*}\right) d x .
$$

The difference to the entropy estimate in the existence proof is that we can now apply the Poincaré inequality to $\rho-\rho^{*}$ to derive the decay of the relative entropy for certain values of $\delta$ :

$$
\begin{aligned}
\frac{1}{\delta} \int_{\Omega}\left(\rho-\rho^{*}\right)\left(c-c^{*}\right) d x & \leq \frac{1}{\delta}\left\|\rho-\rho^{*}\right\|_{L^{2}(\Omega)}\left\|c-c^{*}\right\|_{L^{2}(\Omega)} \leq \frac{1}{2 \delta}\left\|\rho-\rho^{*}\right\|_{L^{2}(\Omega)}^{2}+\frac{1}{2 \delta}\left\|c-c^{*}\right\|_{L^{2}(\Omega)}^{2} \\
& \leq \frac{C_{P}^{2}}{2 \delta}\|\nabla \rho\|_{L^{2}(\Omega)}^{2}+\frac{1}{2 \delta}\left\|c-c^{*}\right\|_{L^{2}(\Omega)}^{2} .
\end{aligned}
$$

Finally, we obtain the entropy estimate

$$
\frac{d}{d t} E^{*}(t)+\left(2-\frac{C_{P}^{2}}{2 \delta}\right)\|\nabla \rho\|_{L^{2}(\Omega)}^{2}+\frac{1}{2 \delta}\left\|c-c^{*}\right\|_{H^{1}(\Omega)}^{2} \leq 0 .
$$

We set $\kappa=\min \left\{1,4 \delta-C_{P}^{2}\right\} /(4 \delta)>0$. Then

$$
\frac{d}{d t} E^{*} \leq-2 \kappa E^{*}
$$

implying the exponential decay of the relative entropy

$$
E^{*}(t) \leq E^{*}(0) e^{-2 \kappa t}, \quad t>0 .
$$

If $\alpha=1$ this immediately implies the desired decay of $\rho-\rho^{*}$ and $c-c^{*}$ in $L^{2}(\Omega)$. For $\alpha=0$, the relative entropy only gives the decay of $\rho-\rho^{*}$ in $L^{2}(\Omega)$.

Step 2: Decay of $c-c^{*}$ for $\alpha=0$. Setting $v^{*}=c^{*}+\delta\left(\rho^{*}\right)^{2}$, we find that

$$
v-v^{*}=c-c^{*}+\delta\left(\rho^{2}-\left(\rho^{*}\right)^{2}\right)=c-c^{*}+2 \delta \rho^{*}\left(\rho-\rho^{*}\right)+\delta\left(\rho-\rho^{*}\right)^{2} .
$$

Replacing $c-c^{*}$ in the elliptic equation for $v$,

$$
0=\Delta v+\rho-c=\Delta v+\left(\rho-\rho^{*}\right)-\left(c-c^{*}\right),
$$

it follows that

$$
0=\Delta\left(v-v^{*}\right)+\left(1+2 \delta \rho^{*}\right)\left(\rho-\rho^{*}\right)+\delta\left(\rho-\rho^{*}\right)^{2}-\left(v-v^{*}\right) .
$$


Hence,

$$
\left\|v-v^{*}\right\|_{H^{1}(\Omega)}^{2}=\left(1+2 \delta \rho^{*}\right) \int_{\Omega}\left(\rho-\rho^{*}\right)\left(v-v^{*}\right) d x+\delta \int_{\Omega}\left(\rho-\rho^{*}\right)^{2}\left(v-v^{*}\right) d x .
$$

To determine the decay of $\left\|v-v^{*}\right\|_{H^{1}(\Omega)}$ we shall derive a uniform bound for $v-v^{*}$. We prove in Step 3 below the boundedness of $\rho-\rho^{*}$ in $L^{\infty}\left(0, \infty ; L^{4}(\Omega)\right)$. Then elliptic regularity for (34) gives $v-v^{*} \in L^{\infty}\left(0, \infty ; H^{2}(\Omega)\right)$ and, by Sobolev embedding, $v-v^{*} \in L^{\infty}\left(0, \infty ; L^{\infty}(\Omega)\right)$. Hence, proceeding with the above estimate and using Young's inequality, we obtain

$$
\left\|v-v^{*}\right\|_{H^{1}(\Omega)}^{2} \leq \frac{1}{2}\left\|v-v^{*}\right\|_{L^{2}(\Omega)}^{2}+C\left\|\rho-\rho^{*}\right\|_{L^{2}(\Omega)}^{2}+\delta\left\|v-v^{*}\right\|_{L^{\infty}(\Omega)}\left\|\rho-\rho^{*}\right\|_{L^{2}(\Omega)}^{2},
$$

such that

$$
\left\|v-v^{*}\right\|_{H^{1}(\Omega)}^{2} \leq 2\left(C+\delta\left\|v-v^{*}\right\|_{L^{\infty}(\Omega)}\right)\left\|\rho-\rho^{*}\right\|_{L^{2}(\Omega)}^{2} \leq C e^{-2 \kappa t} .
$$

This implies for the original unknown $c-c^{*}$ that

$$
\left\|c-c^{*}\right\|_{L^{1}(\Omega)} \leq C\left(\left\|v-v^{*}\right\|_{L^{2}(\Omega)}+2 \delta \rho^{*}\left\|\rho-\rho^{*}\right\|_{L^{2}(\Omega)}+\delta\left\|\rho-\rho^{*}\right\|_{L^{2}(\Omega)}^{2}\right) \leq C e^{-\kappa t} .
$$

Step 3: Proof of $\rho-\rho^{*} \in L^{\infty}\left(0, \infty ; L^{4}(\Omega)\right)$. Using $4\left(\rho-\rho^{*}\right)^{3}$ as a test function in the first equation of (29) and employing the second equation in (29), we infer that

$$
\begin{aligned}
& \partial_{t} \int_{\Omega}\left(\rho-\rho^{*}\right)^{4} d x+12 \int_{\Omega}\left(\rho-\rho^{*}\right)^{2}|\nabla \rho|^{2} d x+8 \delta \int_{\Omega} \rho^{2}\left(\rho-\rho^{*}\right)^{2}|\nabla \rho|^{2} d x \\
&= 12 \int_{\Omega} \rho\left(\rho-\rho^{*}\right)^{2} \nabla \rho \cdot \nabla v d x \\
&= 3 \int_{\Omega} \nabla\left(\rho-\rho^{*}\right)^{4} \cdot \nabla v d x+4 \rho^{*} \int_{\Omega} \nabla\left(\rho-\rho^{*}\right)^{3} \cdot \nabla v d x \\
&= 4\left(1+2 \delta \rho^{*}\right) \rho^{*} \int_{\Omega}\left(\rho-\rho^{*}\right)^{4} d x+\left(3+10 \delta \rho^{*}\right) \int_{\Omega}\left(\rho-\rho^{*}\right)^{5} d x+3 \delta \int_{\Omega}\left(\rho-\rho^{*}\right)^{6} d x \\
&-3 \int_{\Omega}\left(v-v^{*}\right)\left(\rho-\rho^{*}\right)^{4} d x-4 \rho^{*} \int_{\Omega}\left(v-v^{*}\right)\left(\rho-\rho^{*}\right)^{3} d x .
\end{aligned}
$$

The last two terms are bounded by using the nonnegativity of $\rho$ and $v$ and the Cauchy Schwarz inequality:

$$
\begin{aligned}
& -3 \int_{\Omega}\left(v-v^{*}\right)\left(\rho-\rho^{*}\right)^{4} d x-4 \rho^{*} \int_{\Omega}\left(v-v^{*}\right) \rho\left(\rho-\rho^{*}\right)^{2} d x+4\left(\rho^{*}\right)^{2} \int_{\Omega}\left(v-v^{*}\right)\left(\rho-\rho^{*}\right)^{2} d x \\
& \leq 3 v^{*} \int_{\Omega}\left(\rho-\rho^{*}\right)^{4} d x+4 \rho^{*} v^{*} \int_{\Omega} \rho\left(\rho-\rho^{*}\right)^{2} d x+2\left(\rho^{*}\right)^{2} \int_{\Omega}\left(v-v^{*}\right)^{2} d x \\
& \quad+2\left(\rho^{*}\right)^{2} \int_{\Omega}\left(\rho-\rho^{*}\right)^{4} d x .
\end{aligned}
$$

Together with the estimate resulting from (34),

$$
\left\|v-v^{*}\right\|_{H^{1}(\Omega)}^{2} \leq 2\left(1+2 \delta \rho^{*}\right)^{2} \int_{\Omega}\left(\rho-\rho^{*}\right)^{2} d x+2 \delta^{2} \int_{\Omega}\left(\rho-\rho^{*}\right)^{4} d x,
$$


we obtain by interpolation:

$$
\begin{aligned}
& \partial_{t} \int_{\Omega}\left(\rho-\rho^{*}\right)^{4} d x+12 \int_{\Omega}\left(\rho-\rho^{*}\right)^{2}|\nabla \rho|^{2} d x+8 \delta \int_{\Omega} \rho^{2}\left(\rho-\rho^{*}\right)^{2}|\nabla \rho|^{2} d x \\
& \quad \leq C \int_{\Omega}\left(\left(\rho-\rho^{*}\right)^{2}+\left(\rho-\rho^{*}\right)^{4}+\left(\rho-\rho^{*}\right)^{5}\right) d x+3 \delta \int_{\Omega}\left(\rho-\rho^{*}\right)^{6} d x \\
& \quad \leq C(\delta) \int_{\Omega}\left(\rho-\rho^{*}\right)^{2} d x+4 \delta \int_{\Omega}\left(\rho-\rho^{*}\right)^{6} d x .
\end{aligned}
$$

We already know the decay of $\left\|\rho-\rho^{*}\right\|_{L^{2}(\Omega)}$; hence, it remains to bound $\left\|\rho-\rho^{*}\right\|_{L^{6}(\Omega)}$ in terms of the entropy dissipation. To this aim, we use the Gagliardo-Nirenberg with $\theta=d /(d+2)$ and the Young inequality:

$$
\begin{aligned}
& \left\|\left(\rho-\rho^{*}\right)^{3}\right\|_{L^{2}(\Omega)}^{2} \leq C\left(\left\|\nabla\left(\rho-\rho^{*}\right)^{3}\right\|_{L^{2}(\Omega)}^{2 \theta}\left\|\left(\rho-\rho^{*}\right)^{3}\right\|_{L^{1}(\Omega)}^{2(1-\theta)}+\left\|\left(\rho-\rho^{*}\right)^{3}\right\|_{L^{1}(\Omega)}^{2}\right) \\
& \quad \leq C\left\|\rho\left(\rho-\rho^{*}\right) \nabla \rho\right\|_{L^{2}(\Omega)}^{2}+C\left\|\left(\rho-\rho^{*}\right) \nabla \rho\right\|_{L^{2}(\Omega)}^{2}+C\left(\int_{\Omega}\left|\rho-\rho^{*}\right|^{3} d x\right)^{2} \\
& \quad \leq C\left\|\rho\left(\rho-\rho^{*}\right) \nabla \rho\right\|_{L^{2}(\Omega)}^{2}+6\left\|\left(\rho-\rho^{*}\right) \nabla \rho\right\|_{L^{2}(\Omega)}^{2}+C \int_{\Omega}\left(\rho-\rho^{*}\right)^{2} d x \int_{\Omega}\left(\rho-\rho^{*}\right)^{4} d x .
\end{aligned}
$$

The decay $\left\|\rho-\rho^{*}\right\|_{L^{2}(\Omega)} \leq C e^{-\kappa t}$ implies that

$$
\begin{gathered}
\partial_{t} \int_{\Omega}\left(\rho-\rho^{*}\right)^{4} d x+6 \int_{\Omega}\left(\rho-\rho^{*}\right)^{2}|\nabla \rho|^{2} d x+4 \delta \int_{\Omega} \rho^{2}\left(\rho-\rho^{*}\right)^{2}|\nabla \rho|^{2} d x \\
\leq C e^{-2 \kappa t}+C e^{-2 \kappa t} \int_{\Omega}\left(\rho-\rho^{*}\right)^{4} d x,
\end{gathered}
$$

and we conclude that $\rho-\rho^{*} \in L^{\infty}\left(0, \infty ; L^{4}(\Omega)\right)$, which completes the proof.

\section{Numerical Simulations}

This section is intended to illustrate numerically the solutions to the fully parabolic system in two and three space dimensions. We compare the results obtained for $\delta=0$ and $\delta=0.005$ with various values for the exponent $n$ in (2). The simulations were carried out using the COMSOL Multiphysics package with quadratic finite elements. The numerical solutions are for illustration only; a more detailed comparison is the subject of future work. We choose $\Omega=B_{1}(0)$ for simplicity.

The two-dimensional case. We consider the fast-diffusion case $m=\frac{1}{2}$ and prescribe the initial data

$$
\rho_{0}(x, y)=80\left(x^{2}+y^{2}-1\right)^{2}(x-0.1)^{2}+5, \quad c_{0}(x, y)=0 \quad \text { for }(x, y) \in B_{1}(0)
$$

with $M=\int_{\Omega} \rho_{0}(x, y) d x=25 \pi / 3>8 \pi$ (see Figure 2 left). The maximal density is $\rho_{\max }=21.5$. We recall that solutions to the classical parabolic-elliptic Keller-Segel model $(m=1$ and $\delta=0)$ blow up in finite time when the initial mass $M$ is sufficiently large. More precisely, in the radial case, under an additional assumption on the second moment, the solution blows up if $M>8 \pi$ [25] or, in the non-radial case, if $M>4 \pi$ [24]. Since 
$m>1$ leads to global existence results for the parabolic-elliptic system [4, 21], one may conjecture that the cell density of the parabolic-parabolic model blows up in finite time for $M>8 \pi$ if $m<1$. We confirm this conjecture numerically for the case $m=\frac{1}{2}$ and the above initial datum.

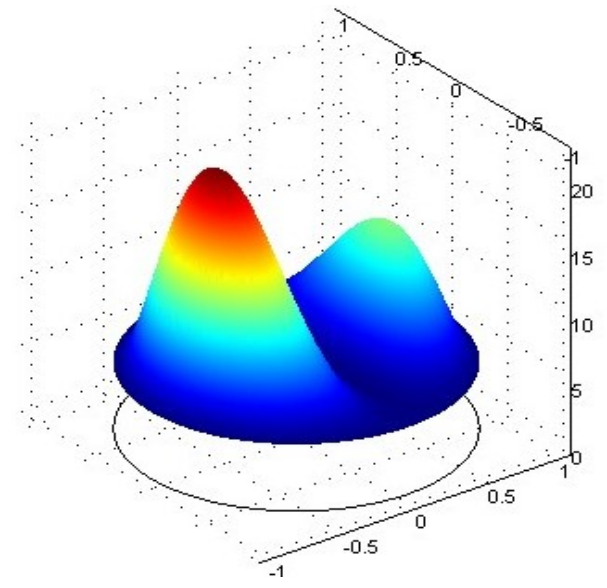

(a) $t=0, \rho_{\max }=21.5$.

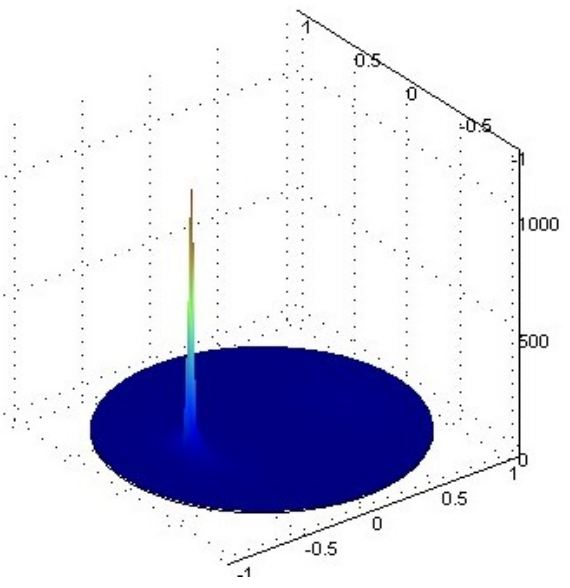

(b) $t \approx 0.15, \rho_{\max } \approx 1212$.

Figure 2. Cell density with $\delta=0$.

The nonlinear diffusion terms cause numerical difficulties whenever the solution becomes close to zero. Indeed, the numerical solution may become negative, and the simulations break down. Clearly, this can be handled by developing a positivity-preserving numerical scheme, similarly as for the porous-medium equation. Since we are using the black-box solver COMSOL Multiphysics, we solve this problem simply by a projection method, i.e., we replace diffusion terms by $\Delta(\max \{\rho, \varepsilon\})^{m}$ with $m=\frac{1}{2}, \varepsilon=0.005$ and $\Delta(\max \{\rho, 0\})^{n}$, respectively.

The cell density of the Keller-Segel model with $m=\frac{1}{2}$ and $\delta=0$ at time $t \approx 0.15$ is depicted in Figure 2 (right). Shortly after that time, the simulations break down which indicates the blow up of solutions. Surprisingly, the singularity forms in the interior of the domain in contrast to the classical Keller-Segel model $(m=1$ and $\delta=0)$ for which blow up occurs at the boundary. Our numerical experiments confirm this behavior for the model with $m=1$ and $\delta=0$ (results are not shown). Thus, the unexpected behavior seems to be an effect of the fast cell diffusion.

Next, we turn to the case $m=\frac{1}{2}$ and $\delta>0$. Figure 3 shows the cell density at time $t=1000$ for various exponents $n=\frac{5}{4}, \frac{11}{8}, \frac{3}{2}, 2$. The solutions have essentially reached their steady state at $t=1000$. Notice that, according to (5), the admissible parameter range for $n$ is $\frac{5}{4}<n \leq \frac{3}{2}$. Although some of the values for $n$ used in the simulations are theoretically not admissible, the solution exists numerically for all time. However, we observed numerical difficulties for large values for $n$ (e.g. $n=6$ ) which may indicate that the upper bound for $n$ in terms of $m$ is more than just a technical assumption. We see 
from Figure 3 that the larger the value of $n$, the more regular the solution becomes, at least in the tested parameter range for $n$.

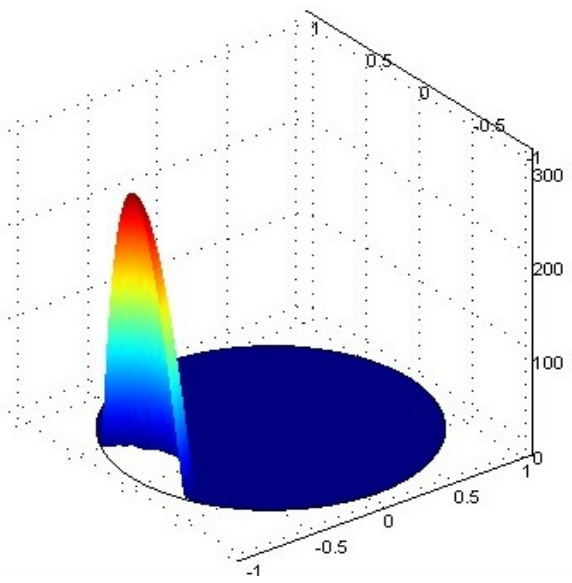

(a) $n=\frac{5}{4}, \rho_{\max } \approx 304$.

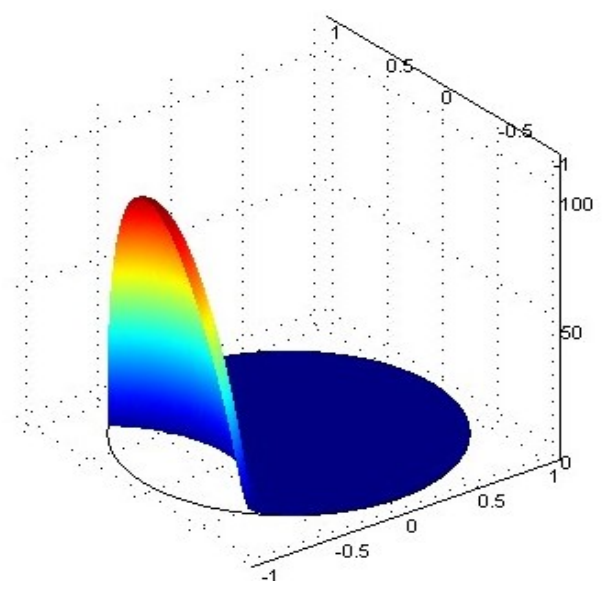

(c) $n=\frac{3}{2}, \rho_{\max } \approx 110$.

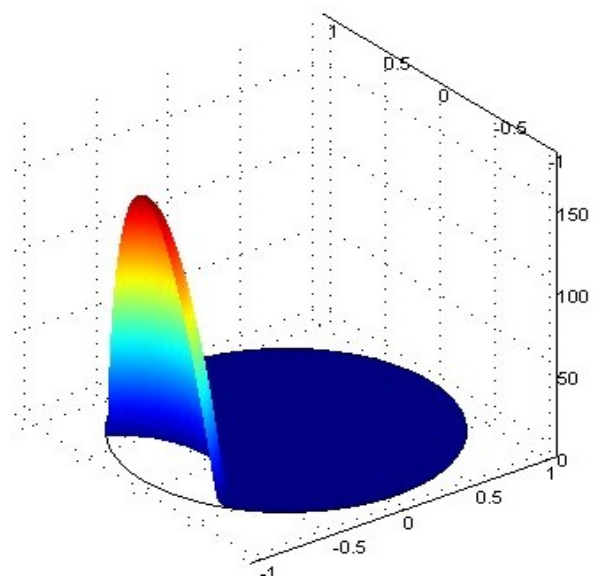

(b) $n=118, \rho_{\max } \approx 174$.

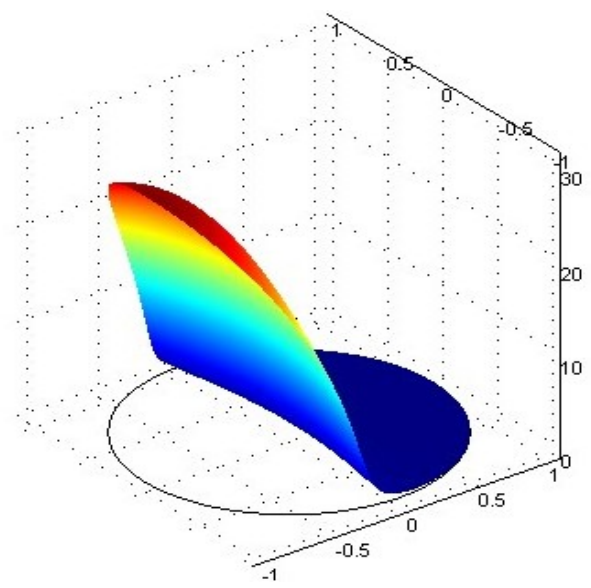

(d) $n=2, \rho_{\max } \approx 30$.

Figure 3. Cell density at time $t=1000$ with $\delta=0.005$.

In the limit of vanishing additional cross-diffusion $\delta \rightarrow 0$, we expect that the solutions converge to the solution to the corresponding Keller-Segel model with $\delta=0$. This is numerically confirmed in Figure 4. For $\delta=0.005$, the cell density reaches its maximum at the boundary (see Figure 3), whereas the maximum is attained in the interior of the domain for very small values of $\delta$. Thus, it seems that the cross-diffusion regularization produces a stationary state which is more concentrated on the boundary.

The three-dimensional case. We consider the linear case $m=1$ and prescribe the initial data

$$
\rho_{0}(x, y, z)=10+80\left(x^{2}+y^{2}+z^{2}-1\right)^{2}(x-0.4)^{2}, \quad c_{0}(x, y, z)=0, \quad(x, y) \in B_{1}(0),
$$




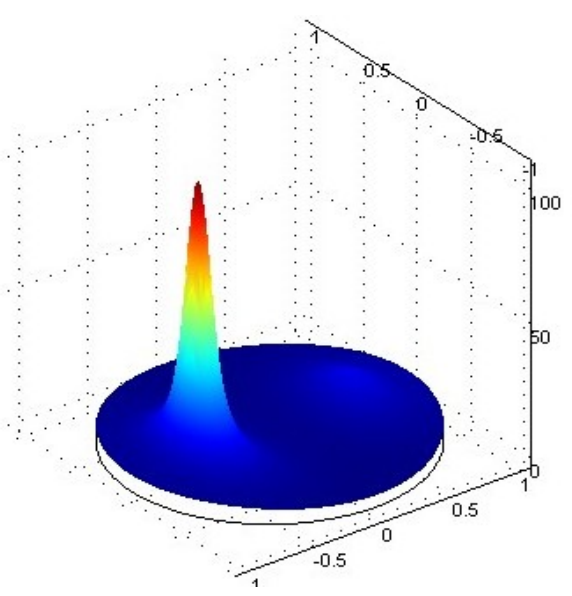

(a) $\delta=10^{-4}, \rho_{\max } \approx 107$.

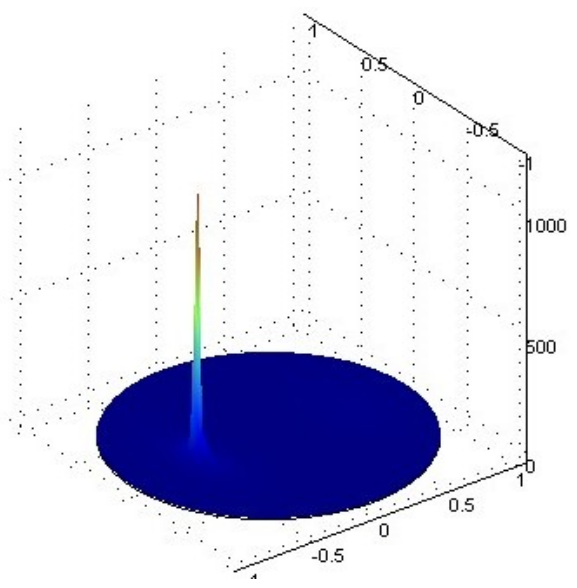

(c) $\delta=10^{-8}, \rho_{\max } \approx 1201$.

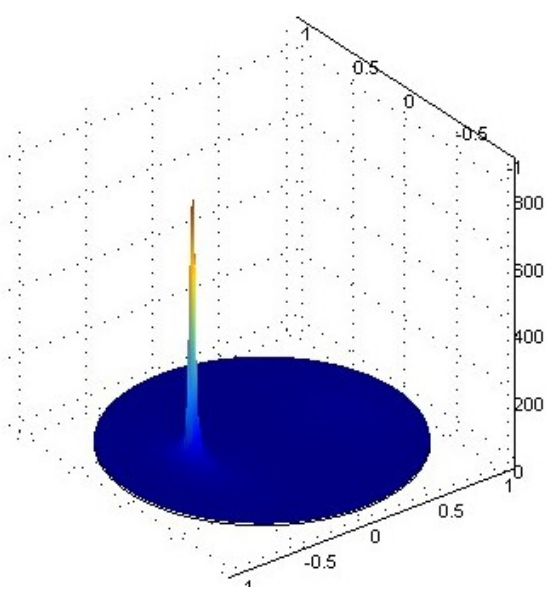

(b) $\delta=10^{-6}, \rho_{\max } \approx 681$.

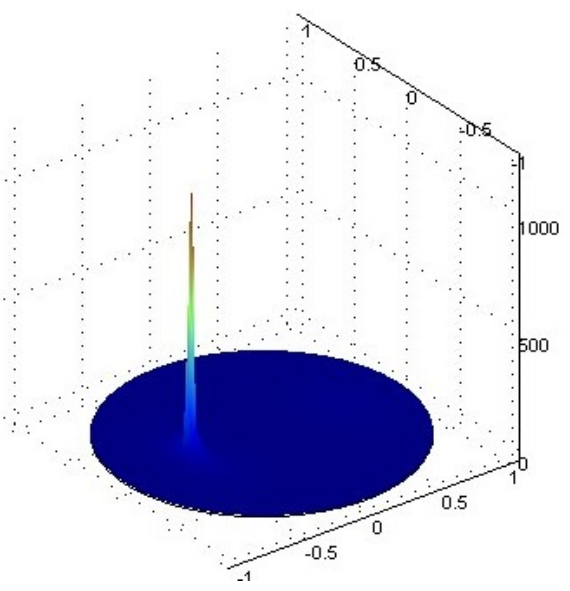

(d) $\delta=0, \rho_{\max } \approx 1212$.

Figure 4 . Cell density at time $t \approx 0.15$ with $n=3 / 2$.

see Figure 5 (left). In three space dimenions, even for the parabolic-elliptic system, there is no critical threshold known for the occurence of blow up. A more complicated functional relation between the second moment and the $L^{3 / 2}$ norm of the cell density has been derived in [7] as a sufficient condition for finite-time blow up. We observed that the numerical solution to the fully parabolic model with $\delta=0$ breaks down after time $t \approx 0.46$, which may indicate a blow-up formation (see Figure 5 right).

The initial data in Figure 5 is represented using slices, since the highest values occur inside the domain, whereas for the following simulations, we use the level-set representation which is more appropriate for demonstrating aggregation phenomena on the boundary.

In Figure 6 we compare the cell density at time $t=1000$ with $\delta=0.005$ and $n=$ $\frac{6}{5}, \frac{13}{10}, \frac{3}{2}, \frac{7}{4}$. At $t=1000$, the solutions have essentially reached the (non-homogeneous) steady state. As we already proved in Proposition 4, when performing the same simulation 


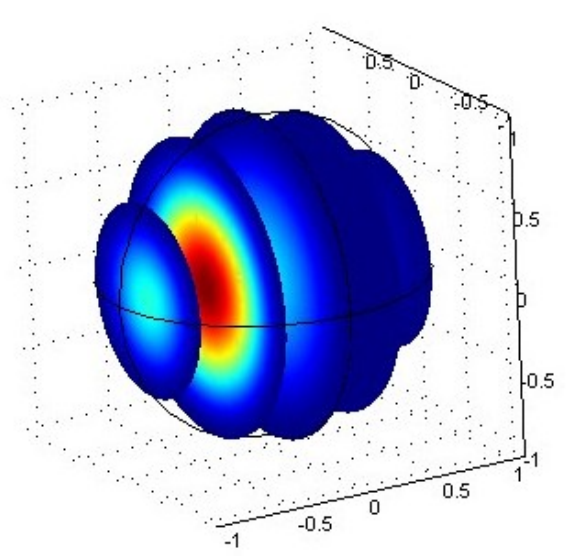

(a) $t=0, \rho_{\max } \approx 46$.

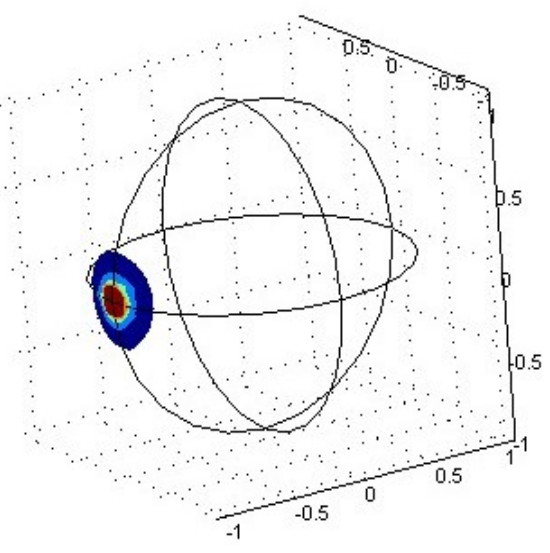

(b) $t \approx 0.46, \rho_{\max } \approx 5460$.

Figure 5. Cell density with $\delta=0$.

with $n=2$, the solution converges to the homogeneous steady state. As in the twodimensional situation, the maximal cell density is achieved at the boundary.

\section{REFERENCES}

[1] P. Biler. Global solutions to some parabolic-elliptic systems of chemotaxis. Adv. Math. Sci. Appl. 9 (1999), 347-359.

[2] A. Bonami, D. Hilhorst, E. Logak, and M. Mimura. A free boundary problem arising in a chemotaxis model. In: Free Boundary Problems, Theory and Applications (Zakopane, 1995). Pitman Res. Notes Math. Ser. 363, pp. 363-373. Longman, Harlow, 1996.

[3] M. Burger, M. Di Francesco, and Y. Dolak-Struss. The Keller-Segel model for chemotaxis with prevention of overcrowding: linear vs. nonlinear diffusion. SIAM J. Math. Anal. 38 (2006), 1288-1315.

[4] V. Calvez and J.A. Carrillo. Volume effects in the Keller-Segel model: energy estimates preventing blow-up. J. Math. Pure Appl. 86 (2006), 155-175.

[5] G. Chavent and J. Jaffre. Mathematical Models and Finite Elements for Reservoir Simulation. NorthHolland, Amsterdam, 1986.

[6] Y.-S. Choi and Z.-A. Wang. Prevention of blow-up by fast diffusion in chemotaxis. J. Math. Anal. Appl. 362 (2010), 553-564.

[7] L. Corrias, B. Perthame, and H. Zaag. Global solutions of some chemotaxis and angiogenesis systems in high space dimensions, Milan J. Math. 72 (2004), 1-28.

[8] P. Degond, S. Génieys, and A. Jüngel. Symmetrization and entropy inequality for general diffusion equations. C. R. Acad. Sci. Paris, Sér. I 325 (1997), 963-968.

[9] M. Di Francesco and J. Rosado. Fully parabolic Keller-Segel model for chemotaxis with prevention of overcrowding. Nonlinearity 21 (2008), 2715-2730.

[10] A. Friedman. Partial Differential Equations. Holt, Rinehart and Winston, Inc., New York, 1969.

[11] P. Grisvard. Elliptic Problems in Nonsmooth Domains. Pitman, Boston, 1985.

[12] T. Hillen and K. Painter. A user's guide to PDE models for chemotaxis. J. Math. Biol. 58 (2009), 183-217.

[13] T. Hillen, K. Painter, and C. Schmeiser. Global existence for chemotaxis with finite sampling radius. Discr. Contin. Dyn. Sys. B 7 (2007), 125-144. 


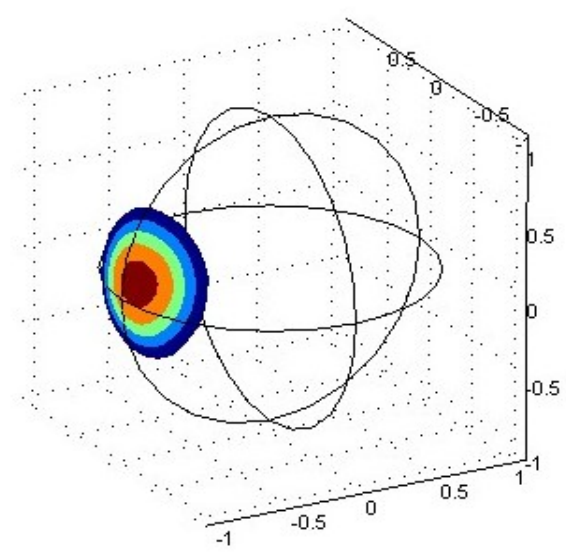

(a) $n=\frac{6}{5}, \rho_{\max } \approx 1023$.

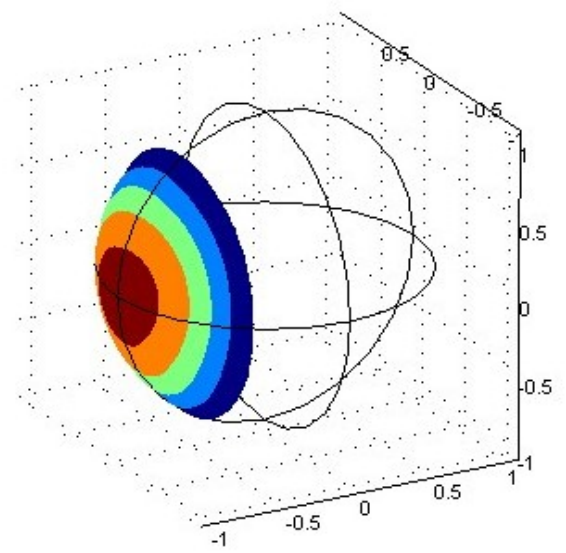

(c) $n=\frac{3}{2}, \rho_{\max } \approx 158$.

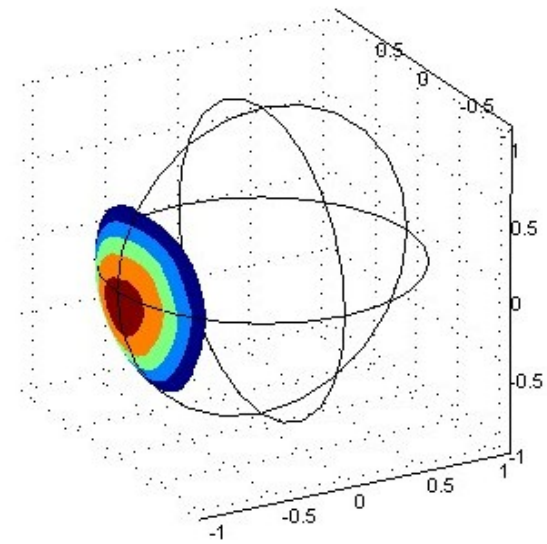

(b) $n=\frac{13}{10}, \rho_{\max } \approx 487$.

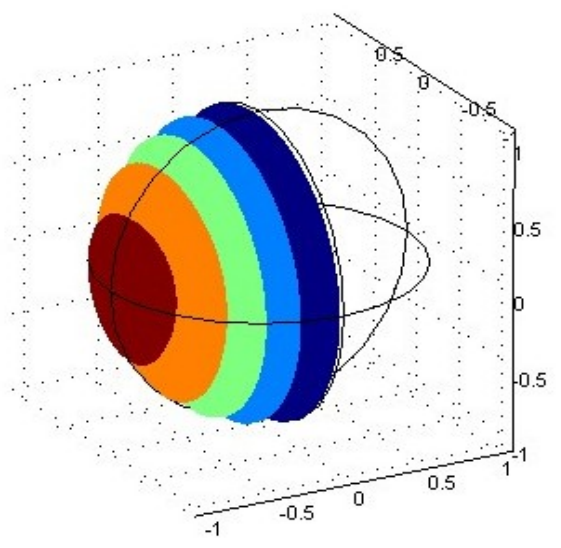

(d) $n=\frac{7}{4}, \rho_{\max } \approx 58$.

Figure 6 . Cell density at time $t=1000$ with $\delta=0.005$.

[14] S. Hittmeir and A. Jüngel. Cross-diffusion preventing blow up in the two-dimensional Keller-Segel model. SIAM J. Math. Anal. 43 (2011), 997-1022.

[15] D. Horstmann. From 1970 until present: The Keller-Segel model in chemotaxis and its consequences I. Jahresber. Deutsche Math. Verein. 105 (2003), 103-165.

[16] D. Horstmann and M. Winkler. Boundedness vs. blow-up in a chemotaxis system. J. Diff. Eqs. 215 (2005), 52-107.

[17] S. Ishida and T. Yokota. Global existence of weak solutions to quasilinear degenerate KellerSegel systems of parabolic-parabolic type. To appear in J. Diff. Eqs., 2011. Online version: doi: $10.1016 / j \cdot j$ de. 2011.02 .012

[18] S. Kawashima and Y. Shizuta. On the normal form of the symmetric hyperbolic-parabolic systems associated with the conservation laws. Tohoku Math. J., II. Ser. 40 (1988), 449-464.

[19] E. Keller and L. Segel. Initiation of slime mold aggregation viewed as an instability. J. Theor. Biol. 26 (1970), 399-415. 
[20] I. Kim and Y. Yao. The Patlak-Keller-Segel model and its variations: properties of solutions via maximum principle. Submitted for publication, 2011. Online version: arXiv:1102.0092.

[21] R. Kowalczyk. Preventing blow-up in a chemotaxis model. J. Math. Anal. Appl. 305 (2005), 566-588.

[22] R. Kowalczyk and Z. Szymańska. On the global existence of solutions to an aggregation model. $J$. Math. Anal. Appl. 343 (2008), 379-398.

[23] J.-L. Lions. Quelques méthodes de résolution des problèmes aux limites non linéaires. Dunod, Paris, 1969.

[24] T. Nagai. Blowup of nonradial solutions to parabolic-elliptic systems modeling chemotaxis in twodimensional domains. J. Inequal. Appl. 6 (2001), 37-55.

[25] T. Nagai, T. Senba, and K. Yoshida. Application of the Trudinger-Moser inequality to a parabolic system of chemotaxis. Funkcial. Ekvac. 40 (1997), 411-433.

[26] C. Patlak. Random walk with persistence and external bias. Bull. Math. Biophys. 15 (1953), 311-338.

[27] J. Simon. Compact sets in the space $L^{p}(0, T ; B)$. Ann. Mat. Pura Appl. 146 (1986), 65-96.

[28] G. Troianiello. Elliptic Differential Equations and Obstacle Problems. Plenum Press, New York, 1987.

[29] M. Winkler. Chemotaxis with logistic source: Very weak solutions and their boundedness properties. J. Math. Anal. Appl. 348 (2008), 708-729.

[30] D. Wrzosek. Model of chemotaxis with threshold density and singular diffusion. Nonlin. Anal. 73 (2010), 338-349.

[31] S. Zheng. Nonlinear Parabolic Equations and Hyperbolic-Parabolic Coupled Systems. Pitman, New York, 1995.

iCREA and Departament de Matemàtiques, Universitat Autònoma de Barcelona, 08193 Bellaterra (Barcelona), Spain. On leave from: Department of Mathematics, Imperial ColLEGE LONDON, LONDON SW7 2AZ, UK.

Department of Applied Mathematics and Theoretical Physics, Wilberforce Road, CamBRIDGE CB3 0WA, UK

Institute for Analysis and Scientific Computing, Vienna University of Technology, Wiedner Hauptstr. 8-10, 1040 Wien, Austria 